\title{
Tebtynis (2020)
}

\section{Claudio Gallazzi et Gisèle Hadji-Minaglou}

\section{OpenEdition \\ Journals}

Édition électronique

URL : https://journals.openedition.org/baefe/2879

DOI : $10.4000 /$ baefe.2879

ISSN : 2732-687X

Éditeur

ResEFE

\section{Référence électronique}

Claudio Gallazzi, Gisèle Hadji-Minaglou, « Tebtynis (2020) » [notice archéologique], Bulletin

archéologique des Écoles françaises à l'étranger [En ligne], Égypte, mis en ligne le 30 mai 2021, consulté le 27 juillet 2021. URL : http://journals.openedition.org/baefe/2879 ; DOI : https://doi.org/10.4000/ baefe. 2879

Ce document a été généré automatiquement le 27 juillet 2021.

\section{(c)}

Le Bulletin archéologique des Écoles françaises à l'étranger est mise à disposition selon les termes de la Licence Creative Commons Attribution - Pas d'Utilisation Commerciale - Pas de Modification 4.0 International. 


\title{
Tebtynis (2020)
}

\author{
Claudio Gallazzi et Gisèle Hadji-Minaglou
}

\section{NOTE DE L'AUTEUR}

Année de la campagne : 2020 (15 septembre - 7 novembre)

Autorité nationale présente : Ayman Ramadan Rateb, inspecteur du Ministère du Tourisme et des Antiquités (MoTA) et responsable du site et de l'entrepôt local des antiquités, a travaillé avec la mission pendant toute la durée de la campagne. Mohamed Ragai Abd el-Hakim et Mohsen Kamal Ahmed ont, à tour de rôle, représenté le MoTA sur le terrain. Mohamed Amin Abd el-Hamid, du département des restaurations du Fayoum, a supervisé les travaux de conservation sur le site du début à la fin de la saison. Iman Alaa El-Din et Mervat Aazer Meseha, inspectrices du MoTA, ainsi qu'Aziza Yehia Othman, Nagwa Mohamed Ahmed, Noha Ali Taha et Hamdi Mohamed el-Sayed, du département des restaurations, ont occasionnellement suivi le travail de certains membres de la mission dans l'entrepôt central des antiquités du Fayoum à Kôm Aushim Numéro et intitulé de l'opération de terrain : 17125 - Fouilles à Umm el-Breigât (Tebtynis)

Composition de l'équipe de terrain : Claudio Gallazzi (papyrologue, Università degli studi di Milano), Gisèle Hadji-Minaglou (archéologue-architecte, Ifao), Sayed Awad Mohamed (archéologue, MoTA), Anna Południkiewicz (céramologue, université de Varsovie), Nikos Litinas (papyrologue, université de Crète), Mohamed Gaber (topographe, Ifao), Ihab Mohamed Ibrahim (photographe, Ifao).

Partenariats institutionnels: Università degli studi di Milano.

Organismes financeurs :

- Ifao

- Università degli studi di Milano

- Ministère des Affaires étrangères et de la coopération internationale d'Italie

1 Notre mission étant investie depuis 1988 dans l'exploration systématique des vestiges de Tebtynis, les fouilles programmées pour 2020 auraient dû être l'extension de celles effectuées en 2019 au nord-est du sanctuaire de Soknebtynis et dans le vaste dépotoir 
situé à l'est de ce dernier'1. Malheureusement, en raison des mesures imposées par la pandémie de Covid-19, une partie des membres de l'équipe n'a pu se rendre en Égypte et la main-d'œuvre locale a dû être fortement réduite par rapport à celle habituellement employée sur le chantier. Ainsi, l'intervention prévue dans le dépotoir a été reportée à 2021 et les fouilles se sont concentrées sur l'habitat au nord-est du sanctuaire. Nous avions déjà travaillé dans ce secteur de 1988 à 1995 et nous y avions repéré une grande rue d'orientation est-ouest, que le contrat démotique de P.Cair. 30617 a (98 av. J.-C.) nomme « dromos de Tefresudj(ty ?) », c'est-à-dire « allée qui conduit au temple du dieu qualifié de Tefresudj(ty?) $»^{2}$. Cette voie, qui débute à l'ouest sur l'esplanade du vestibule du sanctuaire de Soknebtynis, avait alors été suivie sur une longueur de $68 \mathrm{~m}$, mettant au jour de part et d'autre plusieurs bâtiments des périodes ptolémaïque et romaine ${ }^{3}$. En 2019, les fouilles ont repris sur son côté sud, à l'endroit où elles s'étaient arrêtées en 1992, et ont été étendues de plus de $30 \mathrm{~m}$ vers l'est, en direction du temple du dieu dénommé Tefresudj(ty?). Nous avons ainsi dégagé plus d'une dizaine de maisons construites entre le $\mathrm{III}^{\mathrm{e}} \mathrm{s}$. av. et le $\mathrm{II}^{\mathrm{e}} \mathrm{s}$. apr. J.-C., quelques boutiques, une cour abritant plusieurs fours et faisant vraisemblablement fonction de boulangerie, ainsi qu'un podium du II $^{\mathrm{e}} \mathrm{s}$. av. J.-C., tout à fait similaire à ceux qui longent le dromos du temple de Soknebtynis ${ }^{4}$. En septembre 2020, les travaux se sont poursuivis dans la continuité de ceux de l'année précédente, tout d'abord au sud des constructions C4100-I W et C4100-IE, puis à l'est de l'habitation C5100-VE $E^{5}$, couvrant en huit semaines une surface totale de $700 \mathrm{~m}^{2}$ (fig. 1).

Fig. 1. Le secteur fouillé vu du nord (Ihab Mohamed Ibrahim).

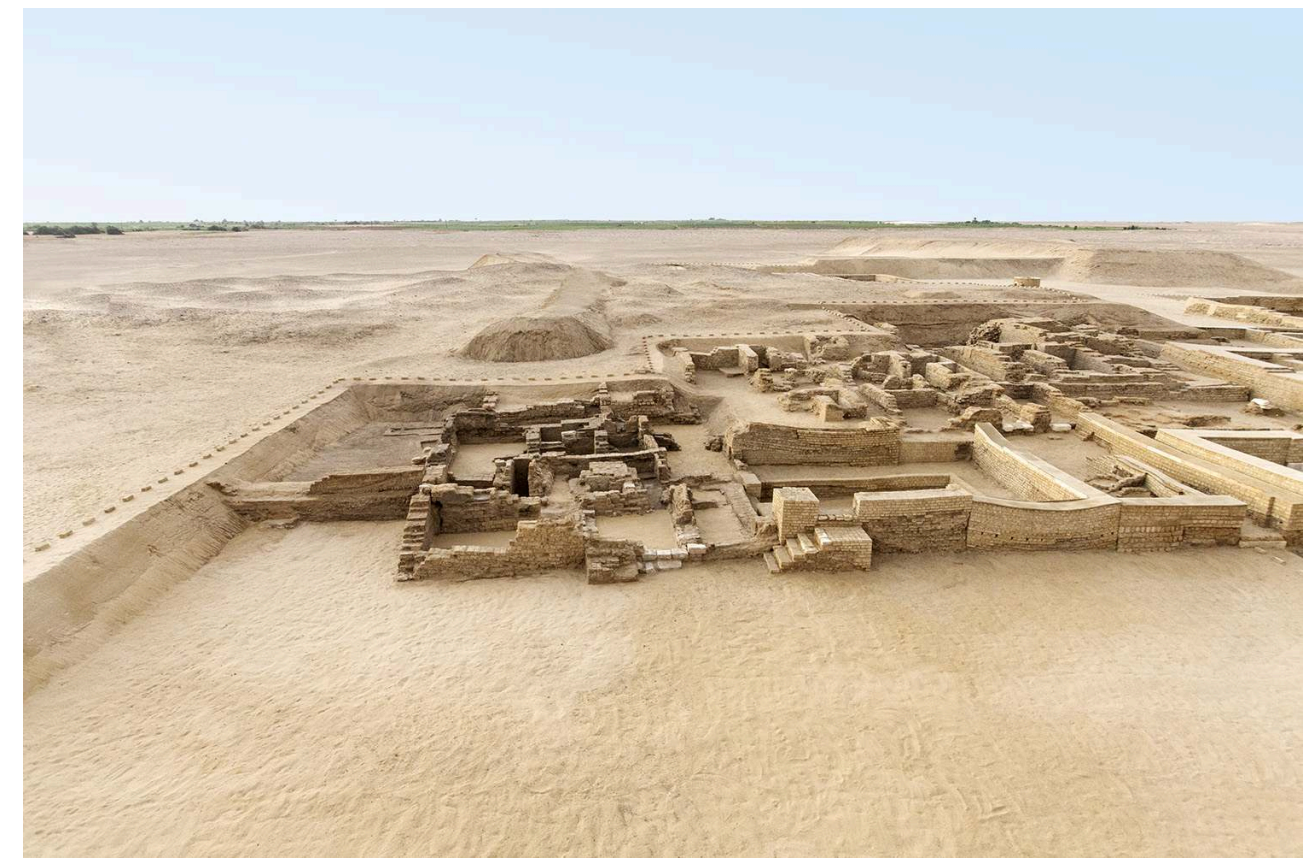

(C) Ifao. 17125_2020_NDMPF_001

\section{L'extension de la fouille vers le sud}

2 La fouille a été étendue au sud de C4100-I W et C4100-I E ${ }^{6}$ car en 2019 était apparu un mur épais, long d'environ $12 \mathrm{~m}$, que l'on pouvait attribuer sans grand risque d'erreur à 
un pyrgos, c'est-à-dire une maison-tour, érigé à l'époque hellénistique. Ce bâtiment occupait l'espace, encore inexploré, compris entre le pyrgos 2400-III mis au jour à l'ouest en $1992^{7}$ et la maison C1100-II dégagée à l'est en $2019^{8}$. La fouille de cet espace allait nous permettre de préciser la topographie du secteur et de déterminer d'éventuelles relations entre le pyrgos déjà exhumé et celui encore enseveli. Ainsi, miseptembre, nous avons entamé l'épaisse couche de surface, constituée de sable, gravats et détritus, en grande partie bouleversée au début du siècle dernier par des chercheurs de papyrus, et nous sommes tombés, contrairement à notre attente, non pas sur une mais sur deux maisons-tours. Les deux pyrgoi sont superposés et ont une emprise quasiment identique, car le bâtiment le plus récent, que nous avons dénommé C2200III, s'est établi sur les fondations du plus ancien, C2200-II, utilisant même une partie des murs qui n'avaient pas été complètement arasés (fig. 2).

Fig. 2. Les pyrgoi C2200-III et C2200-II superposés, vus de l'est (Ihab Mohamed Ibrahim).

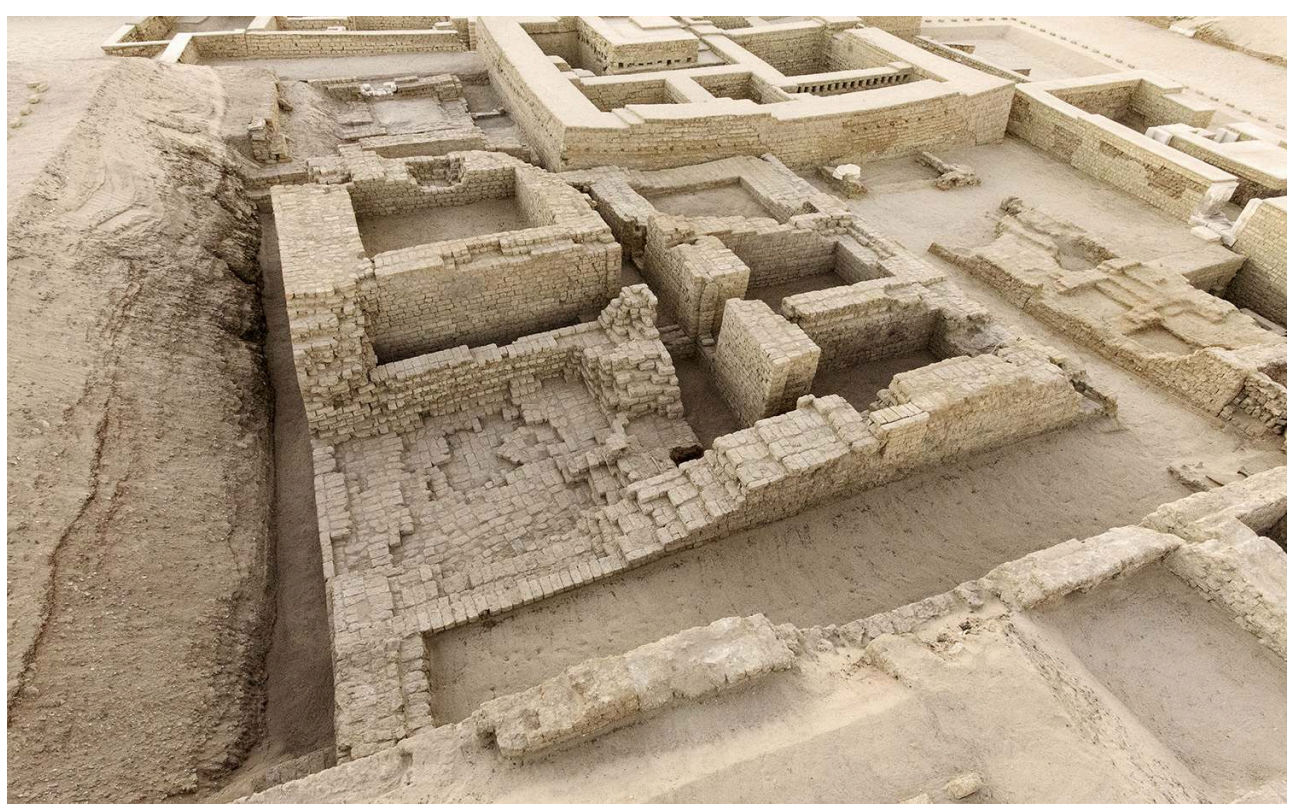

(C) Ifao. 17125_2020_NDMPF_002

C2200-II a été construit vers le milieu du II ${ }^{\mathrm{e}} \mathrm{s}$. av. J.-C. et a duré un demi-siècle. De plan presque carré de 12,25 ( $\mathrm{N}$ et S) × 12,85 (E) × 12,90 (W) $\mathrm{m}$ (fig. 3), il comptait au nord trois pièces non communicantes et s'ouvrant sur un couloir d'orientation est-ouest, long de $9,90 \mathrm{~m}$, large de $1,20 \mathrm{~m}$ et divisé en trois sections, que l'on pouvait fermer par des portes, et au sud une pièce, un couloir nord-sud et un massif d'escalier situé dans l'angle sud-est. Toutes ces pièces sont de tailles très différentes : au nord, la pièce nordouest mesure 3,90 $(\mathrm{N}) \times 3,85(\mathrm{~S}) \times 3,60(\mathrm{E}$ et $\mathrm{W})$, la médiane $2,15(\mathrm{~N}$ et $\mathrm{S}) \times 3,55(\mathrm{E}) \times$ $3,50(\mathrm{~W})$ et celle de l'est $1,80(\mathrm{~N}) \times 1,90(\mathrm{~S}) \times 3,60(\mathrm{E}$ et $\mathrm{W}) \mathrm{m}$; au sud, la pièce sud-ouest a un plan carré de 4,20 $\mathrm{m}$ de côté, le couloir voisin à l'est a pour dimensions $0,90(\mathrm{~N}$ et $\mathrm{S}) \times$ $4,55$ ( $\mathrm{E}$ et $\mathrm{W}) \mathrm{m}$ et le massif de l'escalier occupe un espace de 4,10 ( $\mathrm{N}$ et $\mathrm{S}) \times 4,90$ (E et W) $\mathrm{m}$. Contrairement aux autres, la pièce nord-est a conservé les traces de sa couverture, qui consistait en une voûte à tranches inclinées dont le départ était au nord. Les quelques briques subsistant à sa naissance permettent de restituer sa courbe et de déterminer sa hauteur sous clé, qui était de 1,35 m à partir de l'appui sur les murs est et ouest. La restitution de la hauteur de cette voûte, combinée à la présence dans les 
ruines de plusieurs seuils en bois, nous autorise à établir la hauteur sous clé de la pièce nord-est à environ $2,60 \mathrm{~m}$. Les autres pièces avaient, selon toute vraisemblance, une couverture reposant sur des poutres en bois. La question se pose ici de savoir si les pièces conservées appartenaient au sous-sol ou au rez-de-chaussée de l'édifice. Si nous prenons en considération, même si elles sont plus récentes, deux autres maisons-tours de Tebtynis bien conservées en élévation, à savoir $2400-$ III et $2800^{\circ}$, nous remarquerons que l'une de leurs caractéristiques est l'existence d'un sous-sol occupé par des caves profondes et une entrée située en hauteur. Il en est de même pour un édifice daté de la fin $d u{ } \mathrm{II}^{\mathrm{e}}$ ou du début du $\mathrm{II}^{\mathrm{e}}$ s. av. J.-C., à savoir B1100-I, dont le plan, malgré ses dimensions plus réduites, s'apparente à celui d'une maison-tour ${ }^{10}$. Nous pouvons donc raisonnablement penser que les vestiges conservés sont ceux d'un sous-sol entièrement aménagé en cave. On accédait à ce sous-sol par un escalier relativement étroit qui, compte tenu de la dénivellation restituée grâce à la voûte recouvrant la pièce nord-est, devait compter de 17 à 18 marches réparties sur deux volées. Au sous-sol, devant les premières marches, était creusé un silo, sans doute fermé par une trappe, pour ne pas gêner l'accès à l'escalier. L'arrivée de cet escalier au rez-de-chaussée se trouvait dans l'angle sud-est du couloir nord-sud qui, au sous-sol, était sans doute utilisé comme local de service. Comme pour B1100-I, on accédait à l'entrée de C2200-II par un escalier extérieur dont, malheureusement, aucune trace n'a été trouvée. Toutefois, le plan de B1100-I $\mathrm{I}^{11}$ nous aide à émettre une hypothèse vraisemblable : dans cet édifice, l'entrée à laquelle aboutit l'escalier se trouve en milieu de façade et s'ouvre sur une pièce à partir de laquelle il était facile d'accéder non seulement aux autres pièces, mais aussi à l'escalier intérieur. Dans le cas de C2200-II, cette situation correspond à la pièce médiane nord : si l'escalier se trouvait effectivement à cet endroit, la trace en a disparu derrière le doublage construit à la fin du $\mathrm{II}^{\mathrm{e}}$ ou au début du $\mathrm{I}^{\mathrm{er}} \mathrm{s}$. av. J.-C., au moment de la construction de C2200-III sur les fondations de C2200-II. Un second cas de figure serait une entrée située dans la partie ouest de l'édifice: l'objection principale est l'éloignement de l'escalier intérieur, ce qui est tout à fait inhabituel à Tebtynis. Une troisième hypothèse serait celle d'une situation aux extrémités est ou ouest du couloir est-ouest, mais aucune empreinte d'escalier n'est là aussi visible sur le parement extérieur des murs. L'hypothèse la plus plausible est, par conséquent, celle d'une entrée située en milieu de façade nord. Dans ce cas, l'escalier était établi dans l'espace qui était bordé au nord par la maison contemporaine C8100-II et dont nous ne connaissons pas la limite, car le mur sud de C8100-II a été détruit par les maisons C4100-I W et C4100-I E ${ }^{12}$. À l'ouest, cet espace s'ouvrait sur une rue longeant le mur ouest de C2200-II, qui débouchait au nord sur le dromos de Tefresudj(ty ?). À l'est, il aboutissait à une cour qui appartenait à C2200-II. Celle-ci, avec une largeur variant de $1,85 \mathrm{~m}$ au sud à $2,45 \mathrm{~m}$ au nord, se développait entre le mur est de C2200-II et deux murs contemporains appartenant à des constructions encore sous les sables. 
Fig. 3. Plan du pyrgos C2200-II (sous-sol) et de la maison C4200-II. Milieu du ॥ ${ }^{\mathrm{e}}$ s. av. J.-C. (G. HadjiMinaglou, Mohamed Gaber).

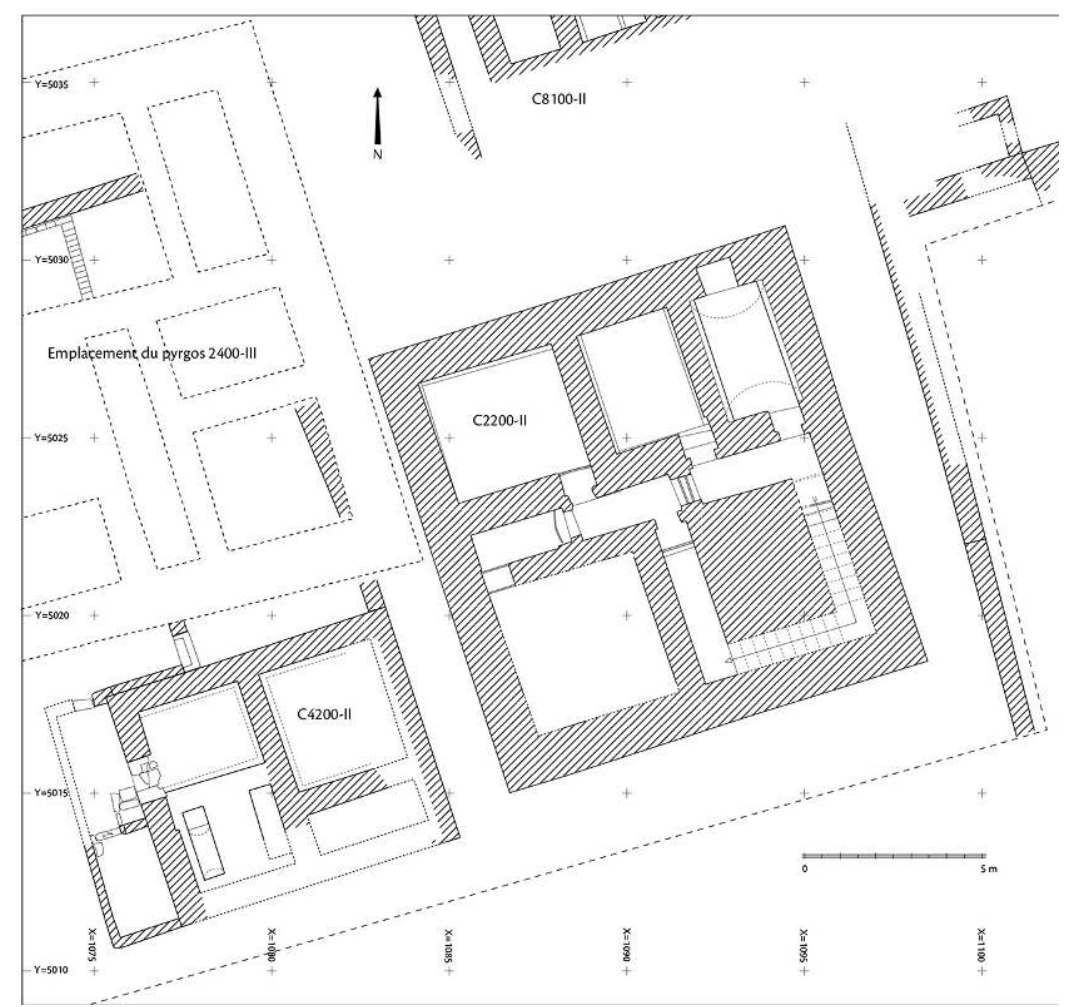

(C) Ifao. 17125_2020_NDMCN_001

C2200-III a un plan identique à celui de C2200-II, mais ses dimensions extérieures diffèrent légèrement, de même que celles de certains espaces intérieurs (fig. 3 et 4). Ces différences résultent de la reconstruction, à la fin du $\mathrm{II}^{\mathrm{e}} \mathrm{s}$. ou au début du $\mathrm{I}^{\mathrm{er}} \mathrm{s}$. av. J.-C., des murs extérieurs dont la largeur est passée de 1,20-1,30 m à 1,60 m. Les murs anciens ont été arasés plus ou moins jusqu'au niveau des fondations, sur lesquelles la nouvelle maçonnerie s'est appuyée tout en débordant, dans le cas de l'angle sud-ouest, en porteà-faux de quelques centimètres vers l'extérieur. Les dimensions extérieures du nouvel édifice sont $12,25(\mathrm{~N}) \times 12,35(\mathrm{~S}) \times 13,25(\mathrm{E}) \times 13,35(\mathrm{~W}) \mathrm{m}$; celles de la pièce sud-ouest ont été réduites à 3,85 $(\mathrm{N}$ et $\mathrm{S}) \times 3,65(\mathrm{E}) \times 3,80(\mathrm{~W}) \mathrm{m}$; la longueur du couloir voisin l'a été de $25 \mathrm{~cm}$ au sud et celle du couloir est-ouest de $30 \mathrm{~cm}$ à son extrémité ouest. Parallèlement, les deux passages dans le couloir est-ouest, l'entrée de la pièce nordouest et celle du couloir nord-sud ont été bouchés. Par ailleurs, la pièce nord-ouest, celle au sud-ouest et le couloir nord-sud ont été remblayés, ainsi que les deux volées de l'escalier. En effet, l'accès à l'escalier qui menait du sous-sol au rez-de-chaussée a été fermé par un mur en L qui s'appuyait d'une part contre le mur extérieur de l'édifice, d'autre part contre le mur sud de la pièce nord-est. Ce mur délimite un puits qui permettait d'accéder du rez-de-chaussée de C2200-III à la pièce nord-est de C2200-II, dont l'usage en cave a été conservé. La pièce médiane nord de C2200-II a, elle aussi, continué d'être utilisée comme cave et on y accédait à partir de la portion de couloir réservée devant son entrée, dans laquelle on descendait du rez-de-chaussée par une trappe. Quant aux deux espaces ouest du couloir, ils étaient sans doute utilisés pour le stockage et accessibles d'en-haut par une trappe. La pièce nord-ouest, remblayée, présentait une stratigraphie très perturbée, qui a néanmoins fourni d'importants 
renseignements. Une couche épaisse et compacte de fumier recouvrant le sol jusqu'au seuil d'entrée montre que C2200-II est resté, à la fin du II $\mathrm{e}$ s. av. J.-C., quelques temps en ruines, ses murs nord et ouest ayant été arasés, et que dans cet espace ont été parqués des bovins. La couche de fumier a été en outre coupée par les tranchées de fondation des murs de C2200-III, qui ont pris appui sur ceux de C2200-II. Le témoin de ces travaux de maçonnerie est une aire de préparation de mortier d'argile entourée de briques cassées, datée de la fin du $\mathrm{II}^{\mathrm{e}} \mathrm{s}$. ou du début du $\mathrm{I}^{\mathrm{er}} \mathrm{s}$. av. J.-C., qui reposait directement sur le fumier. Ces travaux terminés, l'espace a été comblé. Les vestiges de C2200-III sont ceux du sous-sol et ont été arasés bien au-dessous du niveau du rez-de-chaussée, auquel on accédait, comme pour C2200-II, à partir d'un escalier extérieur.

Fig. 4. Plan du pyrgos C2200-III (sous-sol). Fin du $\|^{\mathrm{e}}$ ou début du $\mathrm{I}^{\mathrm{er}}$ s. av. J.-C. (G. Hadji-Minaglou, Mohamed Gaber).

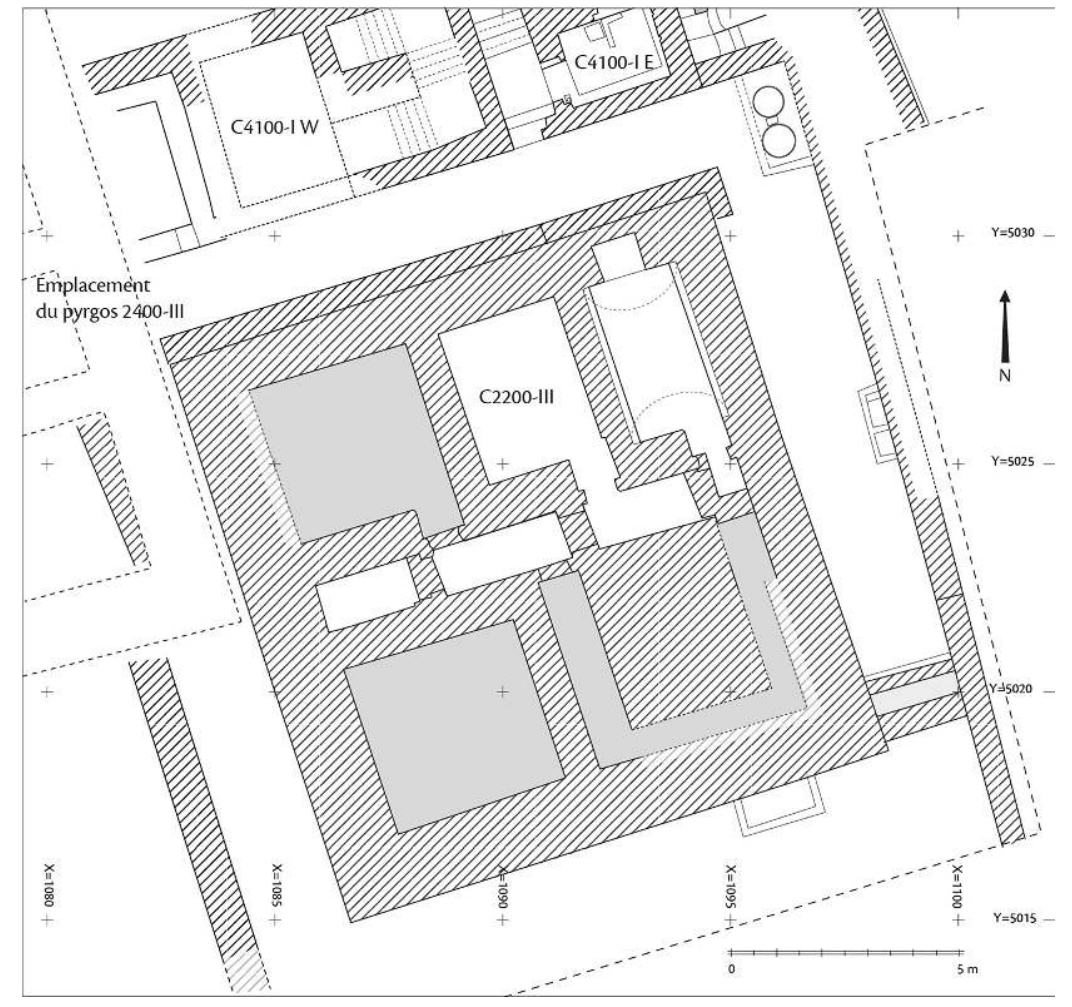

(c) Ifao. 17125_2020_NDMCN_002

C2200-III était bordé sur ses côtés est et nord par une cour, utilisée en commun avec la maison C4100-IE, ainsi que peut-être par C4100-IW, dont nous n'avons malheureusement retrouvé aucune entrée, que ce soit au nord ou au sud, car la moitié ouest de l'édifice a entièrement disparu. Cette cour longeait la totalité du mur est du bâtiment et faisait un coude pour suivre ensuite son mur nord. La section est de la cour, délimitée à l'est par les deux murs qui longeaient déjà C2200-II, avait pour dimensions $2,45(\mathrm{~N}) \times 1,90(\mathrm{~S}) \times 12,10(\mathrm{E}) \times 12(\mathrm{~W}) \mathrm{m}$. À son extrémité sud était installée une grande mangeoire, construite avec soin et dans laquelle du fourrage était encore conservé ; vers le milieu, contre le mur est, était aménagée une banquette double. La section nord de la cour était plus étroite, avec une largeur de $1,75 \mathrm{~m}$. À la rencontre des deux sections, dans l'angle nord-est, se trouvaient deux fours à pain. Un espace, que nous n'avons pas fouillé sur toute sa largeur, s'étendait au sud, avec une banquette disposée 
contre le mur sud du pyrgos. Cet espace était peut-être la cour d'une construction située plus au sud, à moins qu'il ne s'agisse d'une ruelle ou d'une impasse reliée à la ruelle nord-sud longeant C2200-III sur son côté ouest.

C2200-III est resté en usage près d'un siècle et a été désaffecté à la fin du $\mathrm{I}^{\mathrm{er}} \mathrm{s}$. av. J.-C. lorsque le pyrgos 2400-III fut bâti à proximité. Après son abandon, C2200-III a été méthodiquement démantelé et une grande partie de ses briques récupérée au profit de 2400-III, dont les maçons avaient à portée de main un matériau de bonne qualité et prêt à l'usage. Aucune nouvelle construction ne vint s'établir au-dessus des ruines arasées de C2200-III.

7 À l'ouest de C2200-II, de l'autre côté d'une ruelle d'orientation nord-sud large de $1,70 \mathrm{~m}$, se trouvait C4200-II, une petite maison également construite vers le milieu du $\mathrm{II}^{\mathrm{e}} \mathrm{S}$. av. J.-C. (fig. 3). De plan rectangulaire et de dimensions 8,20 ( $\mathrm{N}$ et S) $\times 6,80$ (E et W) $\mathrm{m}$, elle comportait deux pièces au nord, une cage d'escalier au sud-ouest et une troisième pièce au sud-est. L'entrée de l'édifice était à l'ouest, à partir d'une cour, et s'ouvrait sur la pièce nord-ouest qui mesurait $2,90(\mathrm{~N}$ et $\mathrm{S}) \times 2,40(\mathrm{E}$ et $\mathrm{W}) \mathrm{m}$. La pièce voisine à l'est était plus grande : seule la longueur de son côté ouest est certaine et mesure 3,50 m, les dimensions des autres côtés peuvent être restituées à environ $3,20 \mathrm{~m}$ au nord et au sud et 3,50 m à l'est. Des traces sur le sol de ces deux pièces indiquent que la base de leurs murs était protégée par des dalles de pierre. La cage d'escalier occupait une surface restituée de $2,90(\mathrm{~N}) \times 3(\mathrm{~W}) \mathrm{m}$ et avait au sous-sol deux petites caves dont l'une a conservé sa voûte. Les dimensions de la pièce sud-est ne nous sont pas connues avec précision, car le mur sud de la maison se trouve sous les vestiges d'un bâtiment romain qui reste à fouiller, mais il est clair qu'elle n'excédait pas 1,20 m sur ses côtés est et ouest et égalait environ 3,20 m sur les deux autres. La cour était étroite, large d'à peine $1,70 \mathrm{~m}$, et se développait le long du mur ouest de la maison, avec une longueur intérieure de 6,35 $\mathrm{m}$. Elle était divisée en deux parties inégales par un mur situé au sud de l'entrée de la maison, l'espace sud mesurant 1,70 ( $\mathrm{N}$ et $\mathrm{S}) \times 2,70$ (E et $\mathrm{W}) \mathrm{m}$; les murs délimitant l'espace nord ont disparu, à l'exception du montant est de l'entrée, qui se trouvait au nord. Une seconde cour se trouvait au nord de C4200-II. Elle avait une longueur de 5,90 m à partir de l'angle nord-est de la maison et sa majeure partie est située sous le pyrgos 2400-III, de sorte que sa largeur reste indéterminée. Le seuil de son entrée est conservé à l'ouest. Cette seconde cour était sans doute la dépendance d'une maison située plus au nord.

8 Au moment de la construction de C2200-III, à la fin du II ${ }^{\mathrm{e}}$ s. ou au début du $\mathrm{I}^{\mathrm{er}}$ s. av. J.-C., C4200-II était arasée. La partie nord-ouest de ses ruines était recouverte de gravats tassés. Dans le courant du $\mathrm{I}^{\mathrm{er}} \mathrm{s}$. av. J.-C., un mur de direction nord-sud, fondé dans cette couche de gravats, s'est installé 2,20 m à l'ouest de C2200-III. Une épaisse couche de fumier, qui s'est formée à la même époque en relation avec ce mur, indique qu'il s'agissait du mur est d'une cour qui s'étendait vers l'ouest. En effet, 7,90 m plus à l'ouest un mur parallèle et contemporain a été mis au jour en 1995.

9 Les vestiges ayant précédé C4200-II et C2200-II sont très ténus : un mur orienté nordsud remontant à la seconde moitié du $\mathrm{III}^{\mathrm{e}} \mathrm{s}$. av. J.-C. a été trouvé sous le mur est de C2200-II, le long de la ruelle. Plus au nord, une banquette de la même époque est conservée sous la pièce nord-ouest de C2200-II, coupée par le mur ouest de l'édifice. Plus à l'est, sous la cour de C2200-III, moins d'un mètre à l'est du mur est de la construction et parallèle à celui-ci, a été mis au jour un mur construit à la fin du $\mathrm{III}^{\mathrm{e}} \mathrm{s}$. av. J.-C. ou au début du siècle suivant, seul vestige d'un bâtiment qui se développait 
vers l'ouest et qui a été entièrement détruit par C2200-II. Toutes ces bribes de construction ont été fondées sur des couches de détritus jetés pendant la première moitié $\mathrm{du} \mathrm{III}^{\mathrm{e}} \mathrm{s}$. av. J.-C., qui semblent bien appartenir au grand dépotoir qui s'est développé à partir de l'époque hellénistique à l'est du temple de Soknebtynis, entre les dernières constructions de l'agglomération et la nécropole s'étendant dans le désert ${ }^{13}$, les vestiges antérieurs à C2200-II étant, en effet, situés à la lisière du village.

\section{L'extension de la fouille vers l'est, le long du dromos du temple de Tefresudj(ty?)}

10 En prolongeant d'environ $17 \mathrm{~m}$ vers l'est la fouille du dromos du temple de Tefresudj(ty?), un bâtiment dont le mur ouest avait été repéré en 2019 a été mis au jour. Ce bâtiment était le dernier édifice bordant la voie sacrée sur son côté sud. En effet, à cet endroit le dromos débouchait sur une place, sur laquelle se trouvait vraisemblablement l'entrée du temple (fig. 5). Les limites nord, est et sud de cette place ne sont pas encore connues, les bâtiments limitrophes n'ayant pas été atteints. Sa superficie et son aspect ont en outre varié avec le temps. Dans la seconde moitié du $\mathrm{II}^{\mathrm{e}} \mathrm{s}$. av. J.-C., lorsque le podium C5100-III était en activité ${ }^{14}$, l'espace à l'est de celui-ci était libre de constructions, laissant la vue vers le temple dégagée, tandis qu'auparavant le terrain était occupé par les constructions dénommées C3200-I et C3200-II. Lorsque le podium fut désaffecté à la fin du $\mathrm{II}^{\mathrm{e}} \mathrm{s}$. av. J.-C. de nouveaux édifices, C3200-III W et C3200-III E, s'installèrent sur les lieux et la place fut réduite d'une surface de 10,10 × 6,50 m. Il est pour l'instant impossible de préciser les changements dans les limites de l'esplanade au cours des siècles, mais l'extension de la fouille vers l'est, prévue pour la saison prochaine, nous apportera sans aucun doute de nouvelles informations.

Fig. 5. Le dromos du temple de Tefresudj(ty ?) vu de l'est (Ihab Mohamed Ibrahim).

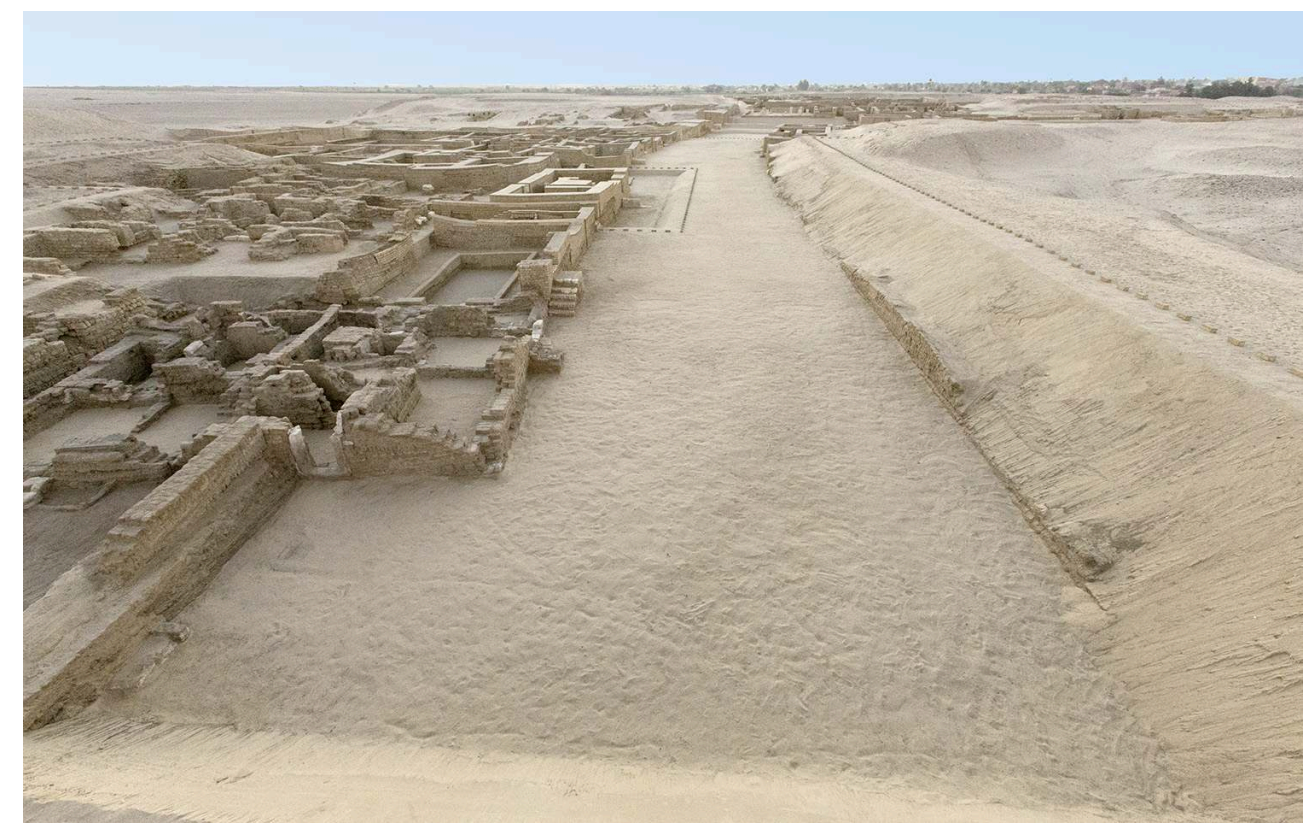

(C) Ifao. 17125_2020_NDMPF_003 


\subsection{Les vestiges d'époque romaine}

11 Le bâtiment situé, à l'époque romaine, à l'angle du dromos et de la place était constitué de deux parties contiguës, C3200-III W, à l'ouest, et C3200-III E, à l'est (fig. 6-9).

C3200-III W a été construit à la fin du $\mathrm{I}^{\mathrm{er}} \mathrm{s}$. av. J.-C. Il avait trois pièces, deux au nord et une au sud. Son entrée était sur le dromos et donnait sur la pièce nord-est. Celle-ci avait une autre porte qui s'ouvrait à l'est, dans un premier temps sur la place ou, plus vraisemblablement, sur une cour, dans un second temps sur C3200-III E. De dimensions $2,35(\mathrm{~N}$ et $\mathrm{S}) \times 2,70(\mathrm{E}$ et $\mathrm{W}) \mathrm{m}$, elle desservait la pièce nord-ouest et un couloir menant à la pièce sud-ouest. La pièce nord-ouest a pour dimensions $1,45(\mathrm{~N}$ et $\mathrm{S}) \times 2,80(\mathrm{E}$ et $\mathrm{W}) \mathrm{m}$ et sa voisine au sud, avec laquelle elle communiquait, 1,45 ( $\mathrm{N}$ et $\mathrm{S}) \times 1,30(\mathrm{E}$ et $\mathrm{W}) \mathrm{m}$. Le couloir est long de 2,50 $\mathrm{m}$ et sur son côté est était aménagé un silo auquel on accédait par deux marches en pierre ; il débouchait au sud sur un passage étroit, long de 2,90 m et large de $48 \mathrm{~cm}$. Ce passage s'ouvrait à l'ouest sur une impasse sur laquelle donnait l'entrée de la cour de la maison contemporaine C5100-IV E ${ }^{15}$. À l'est, il était tout d'abord relié à la cour, puis à C3200-III $\mathrm{E}$.

C3200-III E comprenait au sud-est une pièce rectangulaire de 1,75 $(\mathrm{N}) \times 1,70(\mathrm{~S}) \times 2,80(\mathrm{E}$ et $\mathrm{W}) \mathrm{m}$, où était la porte d'entrée donnant sur la place, et au nord-est une seconde pièce rectangulaire de $3,95(\mathrm{~N}$ et $\mathrm{S}) \times 2,10(\mathrm{E}$ et $\mathrm{W}) \mathrm{m}$, dans le sous-sol de laquelle était enterrée une petite cave voûtée. Ces deux pièces communiquaient par un couloir étroit, large d'à peine $65 \mathrm{~cm}$ et long de $2,15 \mathrm{~m}$, sur lequel donnait un réduit, situé sur son côté sud. À son extrémité ouest, ce couloir aboutissait à la porte permettant d'accéder à la pièce nord-est de C3200-III $\mathrm{W}$ et à un corridor. Ce corridor partait en direction du sud : également étroit (45-55 cm de largeur), il longeait le mur est de C3200-III W sur 2,40 m et rejoignait l'étroit passage menant à l'impasse ouest.

En observant le plan de C3200-III W et de C3200-III E, il apparaît clairement que les deux constructions appartenaient à un même ensemble et, par conséquent, à un même propriétaire. Elles communiquaient entre elles de deux manières, à savoir par la pièce nord-est de C3200-III W et le passage au sud de C3200-III E. Toutefois, leurs plans sont d'une conception différente : C3200-III W était une construction de plan presque carré de $5,70(\mathrm{~N}-\mathrm{S}) \times 5,85(\mathrm{E}-\mathrm{W}) \mathrm{m}$ statiquement autonome, tandis que C3200-III $\mathrm{E}$ a pris appui contre elle. Elle est donc postérieure, sans doute de peu, à C3200-III W : le témoin en est la reprise dans la maçonnerie que l'on observe dans le mur nord à la jonction des deux parties. C3200-III E est par conséquent une extension de C3200-III W, installée sur le terrain qui, du côté de la place, était auparavant occupé par la cour. Le plan du nouvel édifice est rectangulaire et a pour dimensions générales $10,10(\mathrm{~N}) \times 10,30(\mathrm{~S}) \times 6,60(\mathrm{E}) \times$ 5,85 (W) m. Quant à la destination de la construction, C3200-III W a le plan d'une petite maison que l'on a voulu agrandir en lui adjoignant une extension, C3200-III E, qui en doubla la surface. Sa situation à proximité du temple de Tefresudj(ty?) et l'existence de deux accès vers l'extérieur laissent penser qu'il pourrait s'agir d'une maison avec en annexe une boutique ou un atelier. Cette hypothèse serait confirmée par la présence d'un massif rectangulaire aménagé sur le dromos près de la porte d'entrée de C3200III $\mathrm{W}$, vraisemblablement destiné à l'exposition des marchandises.

$\mathrm{Au}$ tout début du $\mathrm{II}^{\mathrm{e}} \mathrm{s}$. apr.J.-C., C3200-III W et C3200-III E ont été partiellement transformés, certains murs ayant été reconstruits sans que, toutefois, le plan général de l'édifice ait radicalement changé. Pour cette nouvelle et dernière phase d'occupation, nous avons désigné la construction par C3200-IV (fig. 10). Le mur séparant les deux 
pièces de C3200-III $\mathrm{E}$ a été irrégulièrement arasé et un nouveau mur a été construit audessus ; à l'ouest, ce mur reposait sur quelques centimètres de l'arase du plus ancien, puis, vers l'est, sur le remblai ayant comblé et recouvert la cave voûtée. Il en a été de même pour le mur sud du passage au sud de C3200-III W, qui, un peu plus étroit, s'est appuyé sur le mur précédent. Le mur de séparation entre les deux pièces nord de C3200-III W a également été rebâti. Le mur est de la pièce nord-ouest a conservé l'empreinte d'une cloison, aujourd'hui disparue, dont l'épaisseur était de $10 \mathrm{~cm}$ et qui séparait deux espaces approximativement de même longueur nord-sud $(85 \mathrm{~cm}$ au nord, $75 \mathrm{~cm}$ au sud). Dans la même pièce, une cloison de même épaisseur tapissait le mur nord ; comme le sol et les murs conservés de la pièce, elle était enduite avec un mortier contenant de la chaux. Les enduits étaient rubéfiés en profondeur et le sol en argile, lui aussi rougi par la chaleur, était couvert d'une couche épaisse d'une vingtaine de centimètres de bois brûlé et de cendres, contenant quelques fragments d'argile rougie ayant conservé l'empreinte de roseaux. Les cendres ont été retrouvées éparpillées jusque dans la petite pièce voisine au sud, dans le couloir du silo et en partie dans le passage au sud. La grande quantité de cendres suggère une activité ayant nécessité un usage continu du feu, celle d'un forgeron par exemple, qui ne nécessite qu'un espace abrité de la lumière du soleil. Malheureusement, aucun outil de travail ne nous est parvenu. Rien de particulier ne caractérise la partie est du bâtiment réaménagé, dont les pièces ont continué d'être utilisées. En revanche, le passage au sud a été bouché à l'ouest, sans doute parce que l'impasse sur laquelle il donnait au $\mathrm{I}^{\mathrm{er}} \mathrm{s}$. de notre ère a été fermée au sud, pour être utilisée comme cour par la maison C5100-V E. Compte tenu des transformations décrites plus haut, il est raisonnable de penser que la moitié est de C3200-IV était utilisée comme habitation, boutique ou les deux à la fois, et que sa partie ouest était réservée à une activité artisanale.

$\mathrm{Au}$ sud de C3200-III W et C3200-III E se trouvait la maison C5200-IV, construite elle aussi à la fin du $\mathrm{I}^{\text {er }}$ s. av. J.-C. (fig. 6-9). De plan rectangulaire et de dimensions 6,30 (N et $\mathrm{S}$ ) × $4,90(\mathrm{E}) \times 4,70(\mathrm{~W}) \mathrm{m}$, elle se composait de trois pièces (deux à l'est, une dans l'angle nord-ouest), d'un couloir recouvrant une cave et d'un réduit. Malheureusement, la stratigraphie des deux pièces est a été bouleversée par une énorme fosse qui a atteint les couches de la fin du $\mathrm{III}^{\mathrm{e}} \mathbf{s}$. av. J.-C. au nord et les vestiges du siècle suivant au sud, détruisant les installations de la maison, y compris une grande partie du mur séparant les deux pièces, au point que l'emplacement du passage menant de l'une à l'autre reste hypothétique. Seule une installation du $\mathrm{I}^{\mathrm{er}} \mathrm{s}$. apr. J.-C. dans l'angle sud-ouest de la pièce sud et quelques couches résiduelles du siècle suivant ont été épargnées. En revanche, à l'exception du remplissage de la cave du couloir et des couches d'abandon, la stratigraphie de la moitié ouest de l'édifice n'a pas été perturbée en profondeur. L'entrée de la maison se trouvait à l'est, sur la place. Elle donnait sur la pièce sud-est, qui mesurait $2,85(\mathrm{~N}) \times 2,75(\mathrm{~S}) \times 1,50(\mathrm{E}$ et $\mathrm{W}) \mathrm{m}$ et qui desservait la pièce nord-est, aux dimensions $2,85(\mathrm{~N}) \times 2,75(\mathrm{~S}) \times 2(\mathrm{E}$ et $\mathrm{W}) \mathrm{m}$, et le couloir, long de $3 \mathrm{~m}$ et large de 0,75 à $1,10 \mathrm{~m}$. Le couloir menait à une cour située à l'ouest; sur son côté nord s'ouvrait la pièce nord-ouest et sur son côté sud se trouvait le réduit. Dans un premier temps, le couloir couvrait une cave, remblayée par la suite, dont la couverture est partiellement conservée : une série de branchages était encastrée dans les fondations des murs nord et sud du couloir et supportait des sections de bois plus courtes, qui étaient ellesmêmes couvertes d'une natte, ou de morceaux de natte, probablement protégée par des briques ou de l'argile aujourd'hui disparues. On pénétrait dans cette cave devant l'entrée de la pièce nord-ouest par une trappe faite de bouts de branchages reliés par 
une cordelette et sur lesquels était fixé un morceau de natte. La pièce nord-ouest était de dimensions réduites, 2 ( $\mathrm{N}$ et $\mathrm{S}) \times 1,35$ ( $\mathrm{E}$ et $\mathrm{W}) \mathrm{m}$, et le mur qui la séparait du couloir était étroit et construit sans soin avec des blocs de pierre récupérés. La cour mesurait $1,45(\mathrm{~N}) \times 1,20(\mathrm{~S}) \times 4,55(\mathrm{E}) \times 4,60(\mathrm{~W}) \mathrm{m}$, son mur ouest s'appuyait contre le mur du passage au sud de C3200-III W, puis de C3200-IV, et son mur sud contre la maison. De sa construction à son abandon, elle abrita des fours à pain : le premier, installé au nord lorsque la maison fut bâtie (fig. 9), un second, construit au-dessus au tout début du II $^{\mathrm{e}} \mathrm{s}$. apr. J.-C., en même temps qu'un troisième, au sud (fig. 10). Au II ${ }^{\mathrm{e}}$ s. apr. J.-C., entre la cour de C5200-IV et la maison C9100-II N ${ }^{16}$ se trouvait une autre cour appartenant à C5100-V E. Elle mesurait 2,50 m sur son côté nord et 3,70 m sur son côté ouest ; les deux autres dimensions peuvent être restituées à $2,80 \mathrm{~m}$ au sud et $3,50 \mathrm{~m}$ à l'est. L'accès à C5100-V E était situé dans l'angle nord-ouest et un four était installé dans l'angle sudest.

17 Au sud de C5200-IV courait une ruelle large de 1,25 m, qui partait de la place et menait à l'impasse sur laquelle donnait l'entrée de la cour de C5100-IV E et, plus tard, à la cour de C5100-V E. Les constructions bordant cette ruelle au sud n'ont pas été fouillées. Sur la place, devant C5200-IV, et dans la ruelle la longeant au sud ont été trouvées des banquettes, qui ne sont pas sans rappeler les installations mises au jour en 2002 sur les bas-côtés du dromos du temple de Soknebtynis ${ }^{17}$.

Fig. 6. Les constructions C3200-III W, C3200-III E, C3200-IV et C5200-IV vues du nord (I. Mohamed Ibrahim).

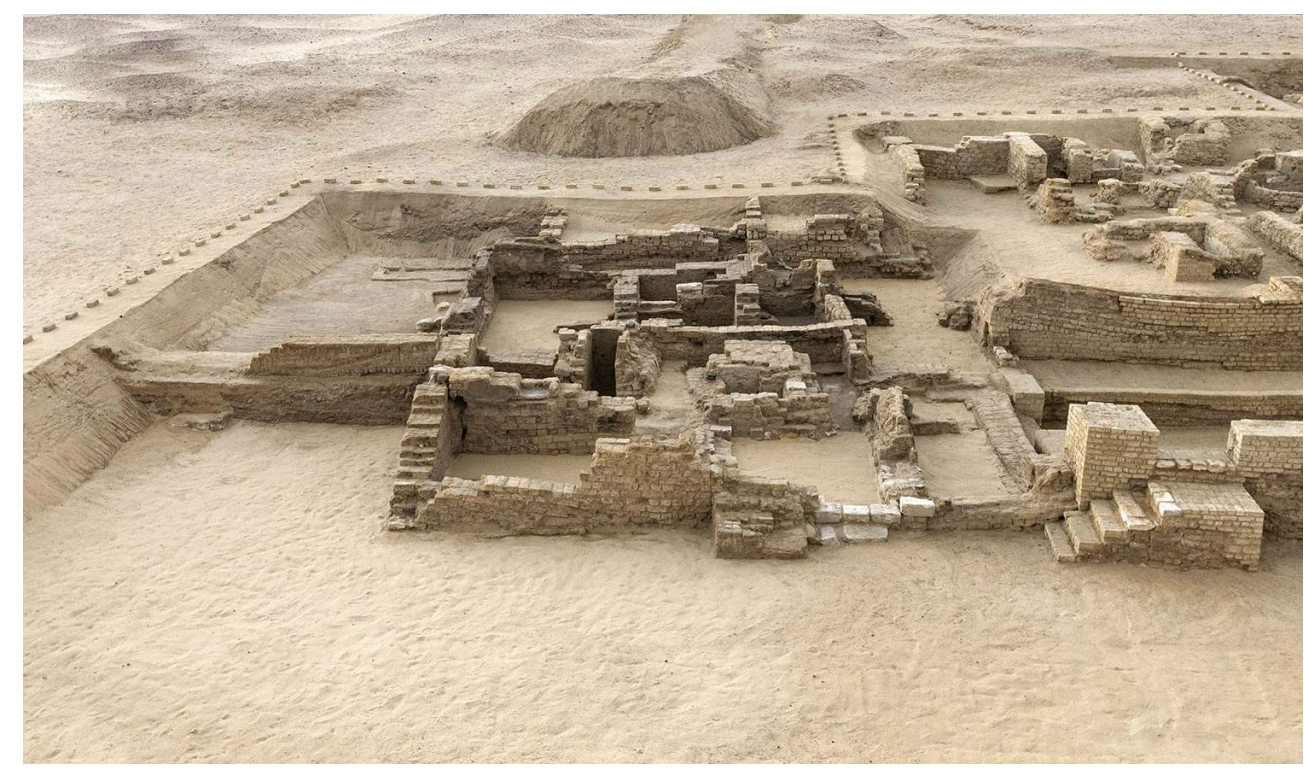

(c) Ifao. 17125_2020_NDMPF_004 
Fig. 7. Les constructions C3200-III W, C3200-III E, C3200-IV et C5200-IV vues de l'est (Ihab Mohamed Ibrahim).

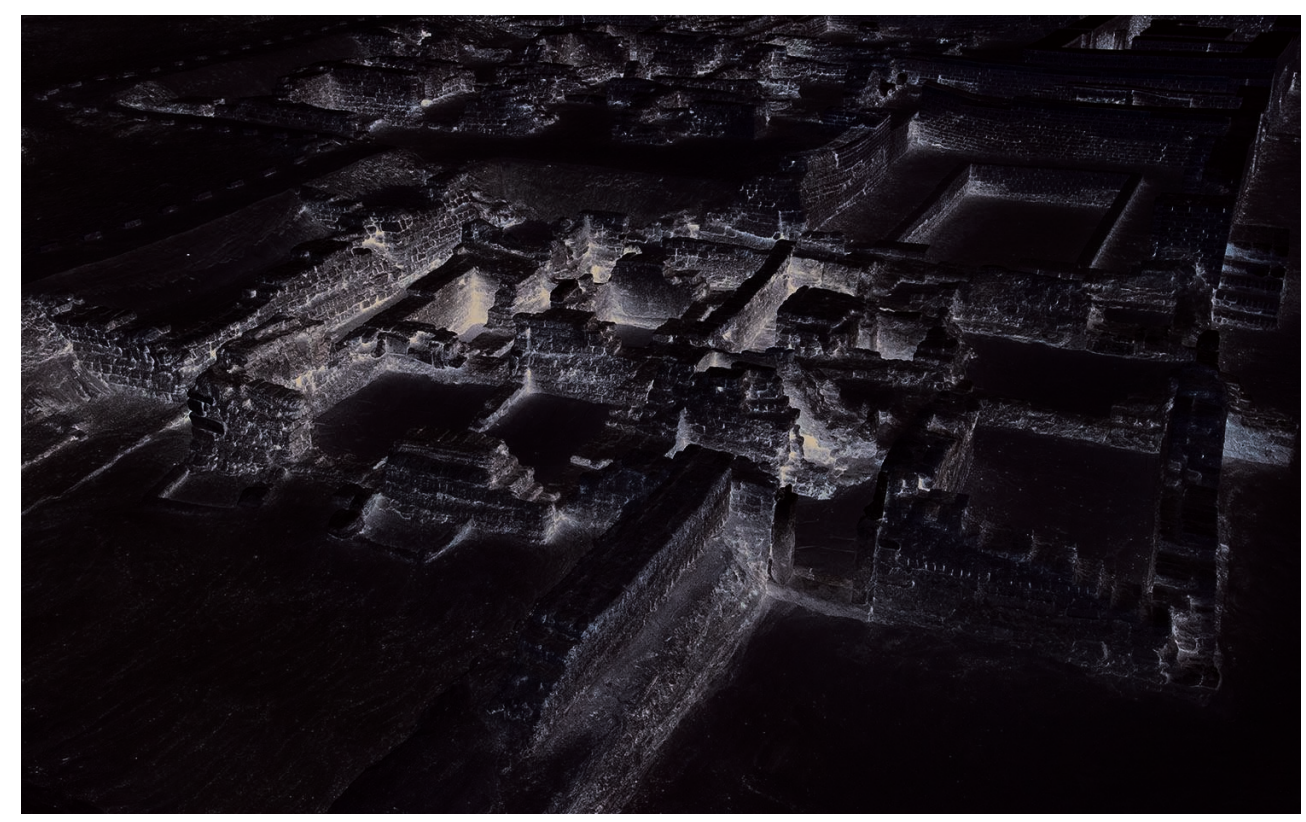

(c) Ifao. 17125_2020_NDMPF_005

Fig. 8. Les constructions C3200-III W, C3200-III E, C3200-IV et C5200-IV vues du sud (Ihab Mohamed Ibrahim).

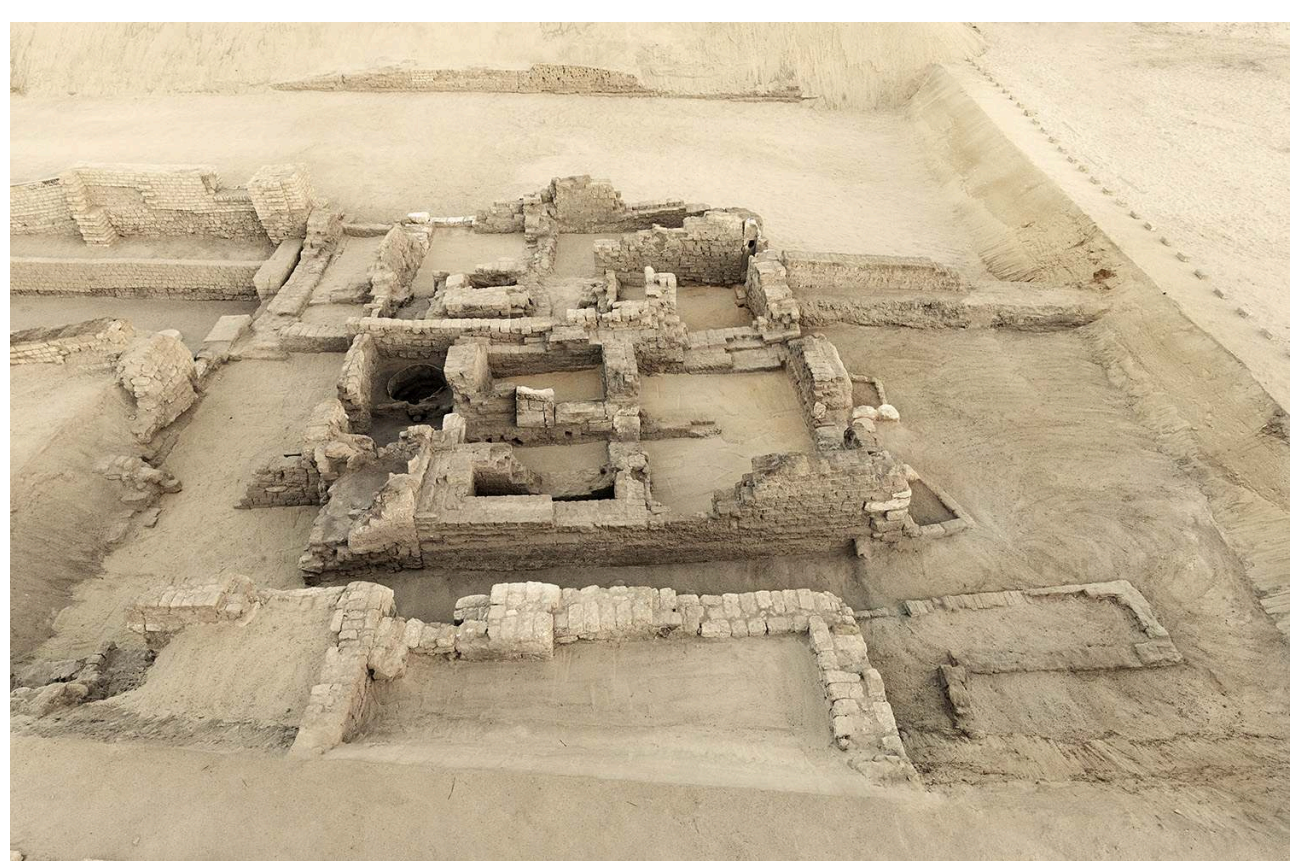

(c) Ifao. 17125_2020_NDMPF_006 
Fig. 9. Plan du secteur est, le long du dromos de Tefresudj(ty ?), au I $^{\text {er }}$ s. apr. J.-C. (G. HadjiMinaglou, Mohamed Gaber).

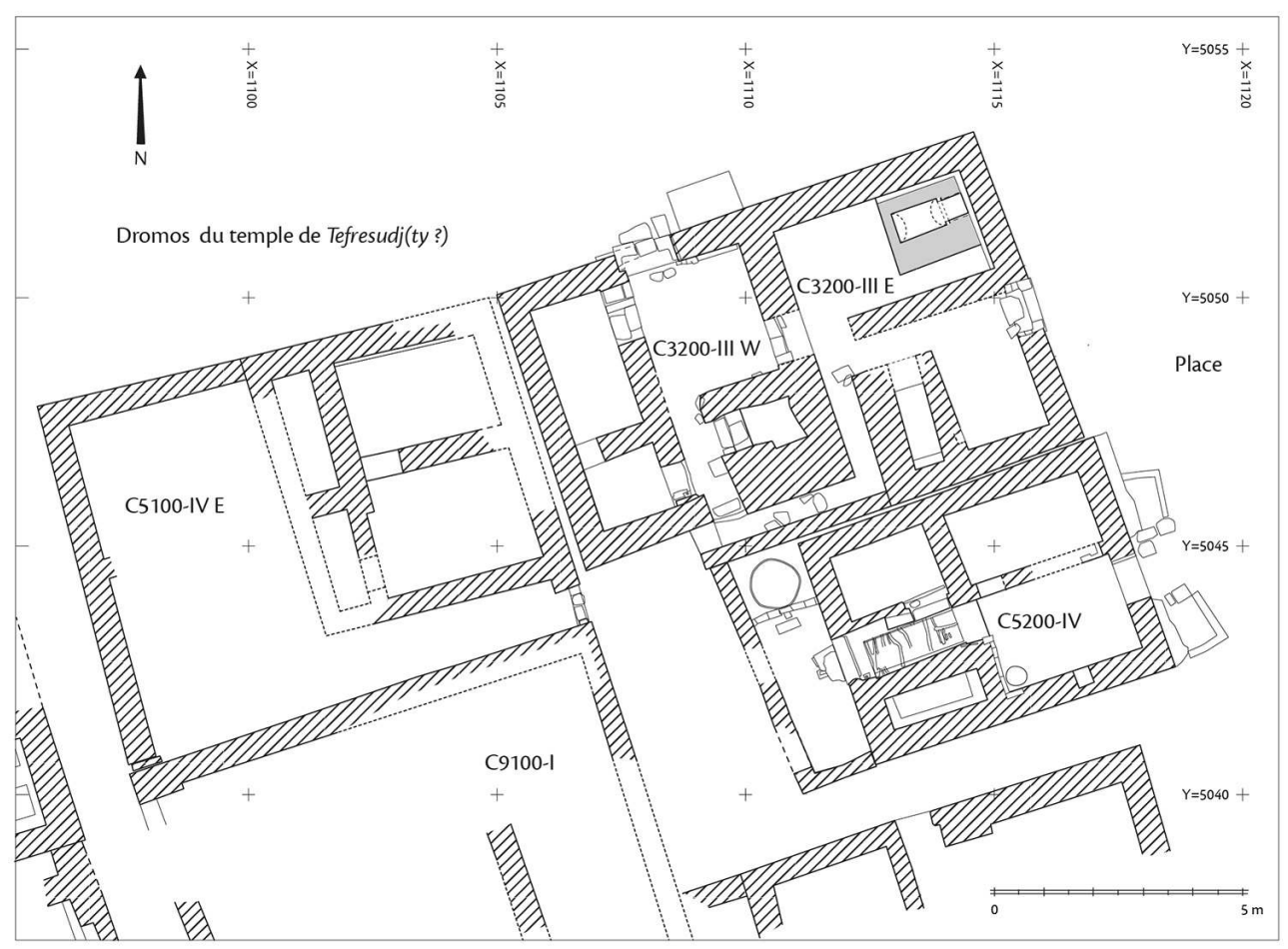

(C) Ifao. 17125_2020_NDMCN_003

Fig. 10. Plan du secteur est, le long du dromos de Tefresudj(ty ?), au ॥ ${ }^{\mathrm{e}}$ s. apr. J.-C. (G. HadjiMinaglou, Mohamed Gaber).

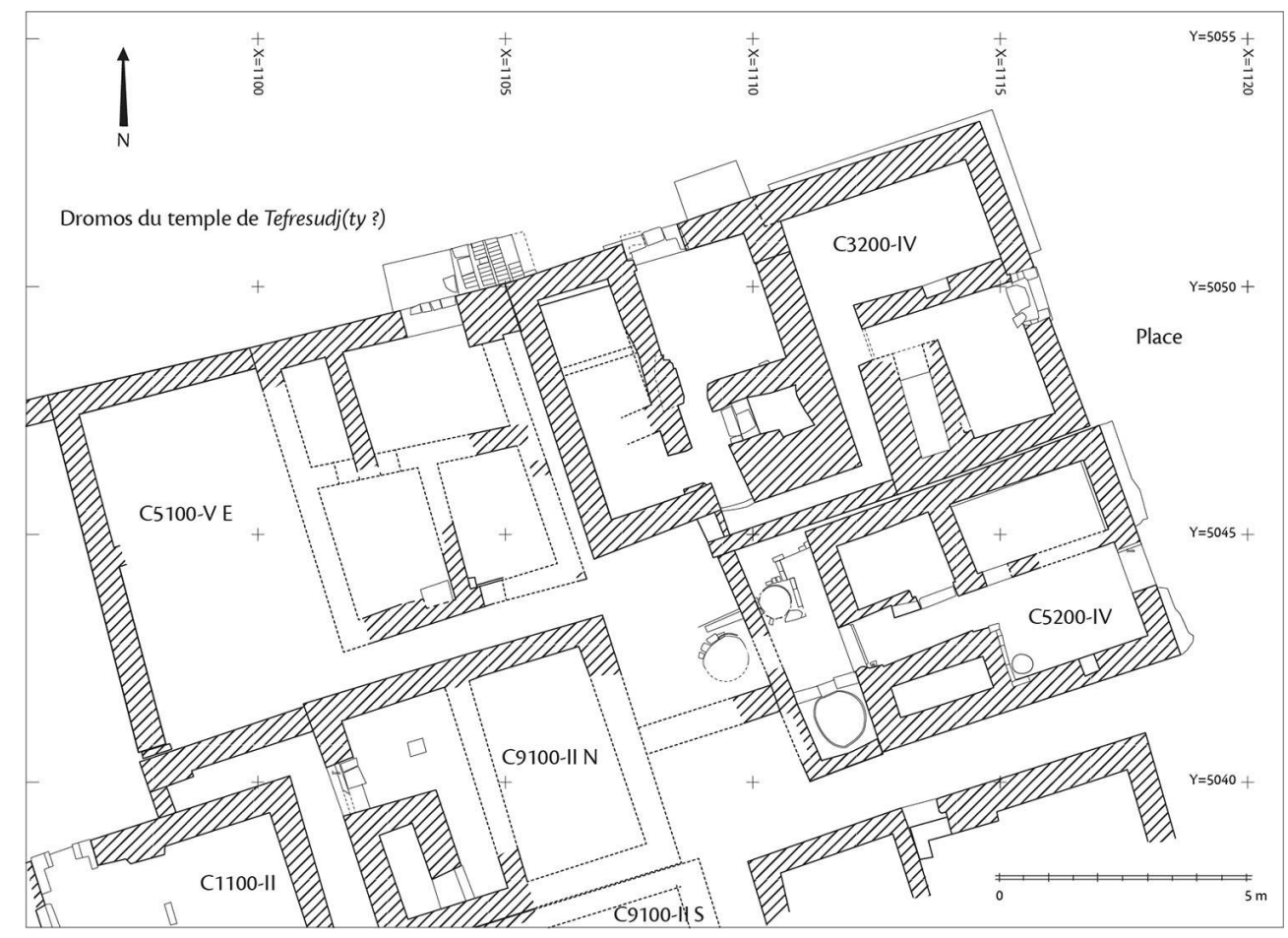

(c) Ifao. 17125_2020_NDMCN_004 


\subsection{Les vestiges ptolémaïques}

18 précédent; ils consistent en quelques bouts de murs situés dans la partie ouest de la zone fouillée. Ceux de la seconde moitié du $\mathrm{III}^{\mathrm{e}}$ s. av. J.-C. sont plus importants. Sous la maison C5200-IV et sous la cour à l'ouest de celle-ci ont été mis au jour des murs que nous avons réunis sous l'appellation C5200-I. Sous C5200-IV, il s'agit de l'angle de deux murs dont l'un est conservé sur toute son épaisseur, $80 \mathrm{~cm}$, tandis que sous la cour subsistent les fondations, larges de $70 \mathrm{~cm}$, de l'angle sud-est d'une construction se développant vers l'ouest, bien qu'aucun mur contemporain n'ait été retrouvé dans cette direction en 2019. Plus au nord, sous C3200-III W et C3200-III E, a été découvert un bâtiment, lui aussi conservé au niveau des fondations. Ce bâtiment, dénommé C3200-I a été repéré sur une surface de $50 \mathrm{~m}^{2}$. Nous n'en connaissons que l'angle nord-est et quelques murs de refend partiellement conservés. Les vestiges de C3200-I suggèrent une construction d'au minimum $9 \mathrm{~m}$ sur son côté nord. Elle était, par conséquent, assez imposante ; c'est également ce que suggère la largeur des murs subsistants, qui étaient d'1 $\mathrm{m}$ pour les murs extérieurs et $0,75 \mathrm{~m}$ pour les murs de refend.

vestiges du il s. av. J.-C. sont mieux conservés que pour le siècle précédent, en particulier pour la seconde moitié du siècle. Dans la première moitié du siècle, le nord du secteur fouillé était occupé par la construction C3200-II dont seules quelques structures légères, telles que des banquettes, ont été repérées. Les ruines mises au jour au sud ne sont guère plus importantes : il s'agit d'un édifice, C5200-II, en grande partie endommagé par C5200-IV, et deux cours, une au nord, l'autre au sud, à l'ouest de l'édifice. Les restes de C5200-II consistent en quelques murs, qui ne nous permettent pas de déterminer l'aspect général du bâtiment. Seule a pu être délimitée une pièce oblongue dont les dimensions, 2,50 $(\mathrm{N}$ et $\mathrm{S}) \times 0,80(\mathrm{E}$ et $\mathrm{W}) \mathrm{m}$, suggèrent que c'était un espace de service ou un couloir en relation avec un escalier qui se trouvait au-dessus. La cour nord lui appartenait peut-être. Elle était adossée au mur est d'une construction se développant plus à l'ouest. Son mur est a disparu sous les bâtiments postérieurs et ses murs sud et nord ne sont pas connus sur toute leur longueur, de sorte que nous n'en avons que la dimension ouest, qui est de 2,95 $\mathrm{m}$. Trois fours y ont été mis au jour, deux ont été construits côte à côte dans l'angle sud-ouest, contre le mur ouest, puis ceux-ci étant arasés, un troisième s'est installé au-dessus d'eux. La cour sud a été presque entièrement détruite par une énorme fosse moderne. Elle a son mur nord en commun avec la cour nord, tandis que son mur ouest est conservé sur moins d'1 m et les autres ont disparu. Un four était établi dans l'angle conservé. Le bâtiment C5200-II et les deux cours à l'ouest de celui-ci ont survécu jusqu'à la fin du II ${ }^{\mathrm{e}}$ s. av. J.-C. Dans la cour nord, un quatrième four a été aménagé contre le mur sud. À la même époque, à l'emplacement de C3200-II, l'espace était libre de constructions (fig. 11). Cet espace ouvert se développait à l'est du podium C5100-III et a sans doute été créé pour permettre à son entrée d'être face au temple de Tefresudj(ty?), sans obstacle. Une fosse de plantation d'arbre dont le diamètre restitué est de $1,40 \mathrm{~m}$, a été retrouvée sur cette esplanade, $7 \mathrm{~m}$ à l'est du podium, avec sa margelle circulaire en briques crues partiellement conservée. 
Fig. 11. Plan du secteur est, le long du dromos de Tefresudj(ty ?), dans la seconde moitié du II $\mathbf{s}$. av. J.-C. (G. Hadji-Minaglou, Mohamed Gaber).

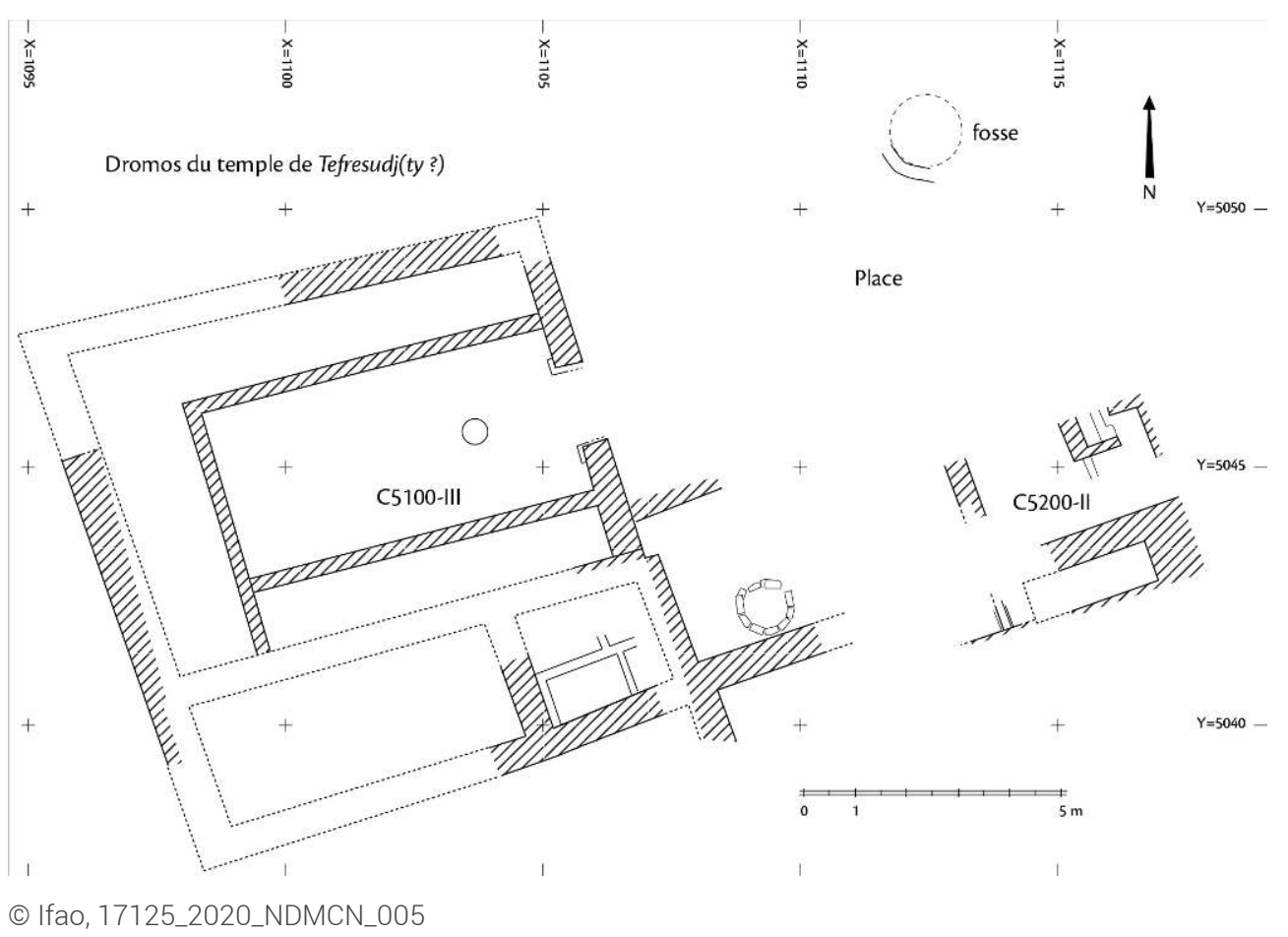

Après son abandon à la fin du $\mathrm{II}^{\mathrm{e}} \mathrm{s}$. av. J.-C. le podium fut arasé, une partie de ses ruines fut remblayée et le reste fut utilisé pour parquer du bétail, jusqu'à ce qu'à la fin du $\mathrm{I}^{\mathrm{er}} \mathrm{s}$. av. J.-C. s'installe au-dessus la maison C5100-IV E ${ }^{18}$. À l'est du podium, les vestiges du $\mathrm{I}^{\mathrm{er}} \mathrm{s}$. av. J.-C. sont très ténus. Les restes de deux fours, détruits par les fondations de C3200-III W ont été découverts sous la pièce nord-ouest de celle-ci en même temps que l'empreinte d'un troisième, tandis que sous la pièce voisine au sud a été mis au jour l'angle sud-est d'une pièce au sol d'argile. Rien n'a subsisté sous C3200-III E, à cause des fondations assez profondes de la construction et de sa cave voûtée, qui ont détruit les structures du $\mathrm{I}^{\mathrm{er}}$ s. av. J.-C. ainsi que la fosse d'arbre du siècle précédent. Une autre fosse de plantation a été creusée sur le dromos de Tefresudj(ty ?), $11 \mathrm{~m}$ au nord-est des ruines du podium : mieux conservée, elle était entourée d'un muret circulaire de 1,60 m de diamètre dont les briques crues ont fondu sous l'effet des eaux d'arrosage versées pendant près d'un siècle. La position de cette seconde fosse laisse penser qu'au $\mathrm{i}^{\mathrm{er}} \mathrm{s}$. av. J.-C., après l'abandon du podium, l'espace investi plus tard par C3200-III E n'était pas libre de constructions ou d'installations, aussi légères que les fours découverts sous la pièce nord-ouest de C3200-III W, pour ne pas avoir laissé de traces.

Peu d'éléments du $\mathrm{I}^{\mathrm{er}}$ s. av. J.-C. situés sous C5200-IV, eux aussi très fragmentaires, ont échappé à la destruction. Ces vestiges, regroupés sous l'appellation C5200-III, consistent en quelques bribes de murs auxquels sont associés des restes de banquettes, mais aussi en une cour. Celle-ci était appuyée contre le mur ouest d'un édifice nommé C9100-I, repéré en $2019^{19}$, mais son plan, bien qu'incomplet, suggère qu'elle était rattachée à C5200-III. Son mur sud a été construit au-dessus de celui de la cour de la seconde moitié $\mathrm{du} \mathrm{II}{ }^{\mathrm{e}} \mathrm{s}$. av. J.-C. et, contrairement à cette dernière, son mur est a été conservé. Il est ainsi possible de déterminer sa largeur est-ouest, qui était de $2,80 \mathrm{~m}$. Nous ne connaissons pas sa longueur totale du nord au sud, mais nous pouvons dire qu'elle était 
d'au moins 3,65 m. Dans l'angle sud-est de la cour ont successivement été installés deux fours à pain et au nord, probablement contre le mur de clôture, aujourd'hui occulté ou détruit par les vestiges plus récents, se trouvait une plateforme pavée de briques crues.

Les fours situés contre les ruines du podium et les quelques restes de banquettes conservées plus à l'est laissent penser qu'au $\mathrm{I}^{\mathrm{er}} \mathrm{s}$. av. J.-C. le secteur était une zone artisanale occupée par des boulangeries et autres installations au service des pèlerins se rendant au temple de Tefresudj(ty?). C'était aussi probablement le cas dans la première moitié du II ${ }^{\mathrm{e}} \mathrm{s}$. av. J.-C. avant que ne soit construit le podium.

\section{Le matériel recueilli}

Aussi bien le long du dromos de Tefresudi(ty ?) que dans l'espace plus au sud occupé par C2200-II, C2200-III et C4200-II, les travaux ont permis de récupérer un certain nombre d'objets. À vrai dire, la moisson des trouvailles a été moins abondante qu'en 2019, car les couches supérieures avaient déjà été entamées au siècle dernier, les bâtiments avaient été en partie vidés et démontés à une époque ancienne, de même que dans la rue et la place aucune grande fosse remplie de tessons et d'objets cassés comparable à celle de l'année précédente n'a été trouvée ${ }^{20}$. Néanmoins, des pièces rares, voire rarissimes, ou exhumées pour la première fois des vestiges de Tebtynis ont été recueillies. Par exemple, parmi les terres cuites, peu nombreuses et toutes fragmentaires, on remarque une Isis-Aphrodite anadyomène presque complète. La statuette, récupérée dans une couche remontant à la deuxième moitié du $\mathrm{I}^{\mathrm{er}} \mathrm{s}$. apr. J.-C., représente la déesse levant les bras pour écarter de son visage ses longs cheveux, le torse et le ventre nus jusque sous le nombril et les jambes recouvertes d'une draperie aux plis disposés en biais et nouée sur le devant. Elle est donc proche des terres cuites présentant le même sujet, connues par ailleurs ${ }^{21}$. Toutefois, un détail la distingue des autres : un enfant en bas âge se tient debout à gauche de la déesse et s'agrippe à son vêtement. Ce petit personnage est inhabituel sur les figurines similaires, mais nous le retrouvons sur une statuette du Musée égyptien du Caire enregistrée sous le $n^{\circ} 63552$ du Journal d'entrée ${ }^{22}$. Ce n'est pas un effet du hasard, car la pièce du Caire a elle aussi été récupérée à Tebtynis, en 1934, dans une maison à l'ouest du dromos principal ${ }^{23}$. Par conséquent, l'enfant d'un côté rend notre terre cuite remarquable du point de vue de la typologie, de l'autre il apporte des indications utiles pour la connaissance de la production et du commerce des statuettes. Il en est de même pour les lampes. Nous n'avons récupéré qu'une douzaine d'exemplaires complets ou presque complets, mais l'un d'eux appartient à un type jusqu'à présent absent à Tebtynis ou dans le Fayoum (fig. 12). Fabriquée en argile beige clair, la lampe a très vraisemblablement été importée en Égypte dans la seconde moitié du $\mathrm{I}^{\mathrm{er}} \mathrm{s}$. av. J.-C. Elle est pourvue d'un bec court, aux côtés concaves décorés de volutes, et présente un réservoir rond avec un médaillon audessus. Celui-ci est bordé de filets, qui se reproduisent au centre, autour du trou de remplissage. Entre les deux séries de filets se déroule une bande moulurée, où sont représentées deux branches liées à leur l'extrémité par un ruban. Chacune de ces branches porte une grenade avec deux oiseaux disposés de part et d'autre du fruit. Trois d'entre eux picorent le fruit, tandis que le quatrième regarde derrière lui ${ }^{24}$. Les faïences ont été également peu abondantes; néanmoins, parmi les pièces récoltées se trouvent des amulettes apparues pour la première fois ou très peu représentées dans le matériel récupéré à Tebtynis : un crocodile soulevant la tête, la queue tournée vers la 
droite, remontant au $\mathrm{III}^{\mathrm{e}} \mathrm{s}$. av. J.-C. ${ }^{25}$ et deux têtes de Bès datées de la même époque, hautes de 4 et $4,5 \mathrm{~cm}$ et pourvues d'un conduit horizontal long de $2 \mathrm{~cm}$, où passait le fil pour les suspendre ${ }^{26}$ (fig. 13). Du petit lot de vanneries il faut sortir et mettre en évidence au moins une natte assez bien conservée, large d'environ $70 \mathrm{~cm}$ et longue de plus de $2 \mathrm{~m}$ ( $\mathrm{II}^{\mathrm{e}} \mathrm{s}$. apr. J.-C.) et un grand panier au fond rectangulaire, qui devait être utilisé pour transporter des denrées à dos d'âne ou de dromadaire (III ${ }^{\mathrm{e}} \mathrm{s}$. av. J.-C.) ${ }^{27}$. Parmi les ustensiles en bois, le plus souvent réduits à des morceaux cassés difficiles à reconnaître, on remarque immédiatement une masse en bois presque intacte datée du $\mathrm{II}^{\mathrm{e}}$ s. apr. J.-C., semblable à celles décrites et reproduites dans PETRIE 1917, p. 40, pl. XLVI, $\mathrm{n}^{\text {os }} 72-73$, et коSACK 1974, p. 21, n ${ }^{\circ} \mathrm{D} 2$, Ph. $\mathrm{D} 2$ : c'est la première découverte à Tebtynis, où auparavant les fouilles n'avaient fourni que des petits maillets comme celui publié dans Milano in Egitto 2017, p. 108, $\mathrm{n}^{\circ} 2.21$. Pour ce qui concerne les morceaux de tissu et les restes de vêtements, rappelons qu'ils ont été peu nombreux, parce que les détritus du dépotoir, dans lesquels ils sont abondants, n'ont pas été fouillés. Malgré cela, les couches du II ${ }^{\mathrm{e}} \mathrm{s}$. apr. J.-C. ont livré deux pièces qui doivent être mentionnées pour leur rareté : un bonnet conique en feutre identique à ceux que nous voyons sur certaines terres cuites, par exemple celles de BRECCIA 1934, p. 56, $\mathrm{n}^{\circ} 387$, pl. XCVIII, $\mathrm{n}^{\text {os }} 547-550$, et un autre en laine multicolore, qui était destiné à un nourrisson ou à un enfant en bas âge (fig. 14).

Fig. 12. Lampe. Seconde moitié du I er s. av. J.-C. (Ihab Mohamed Ibrahim).

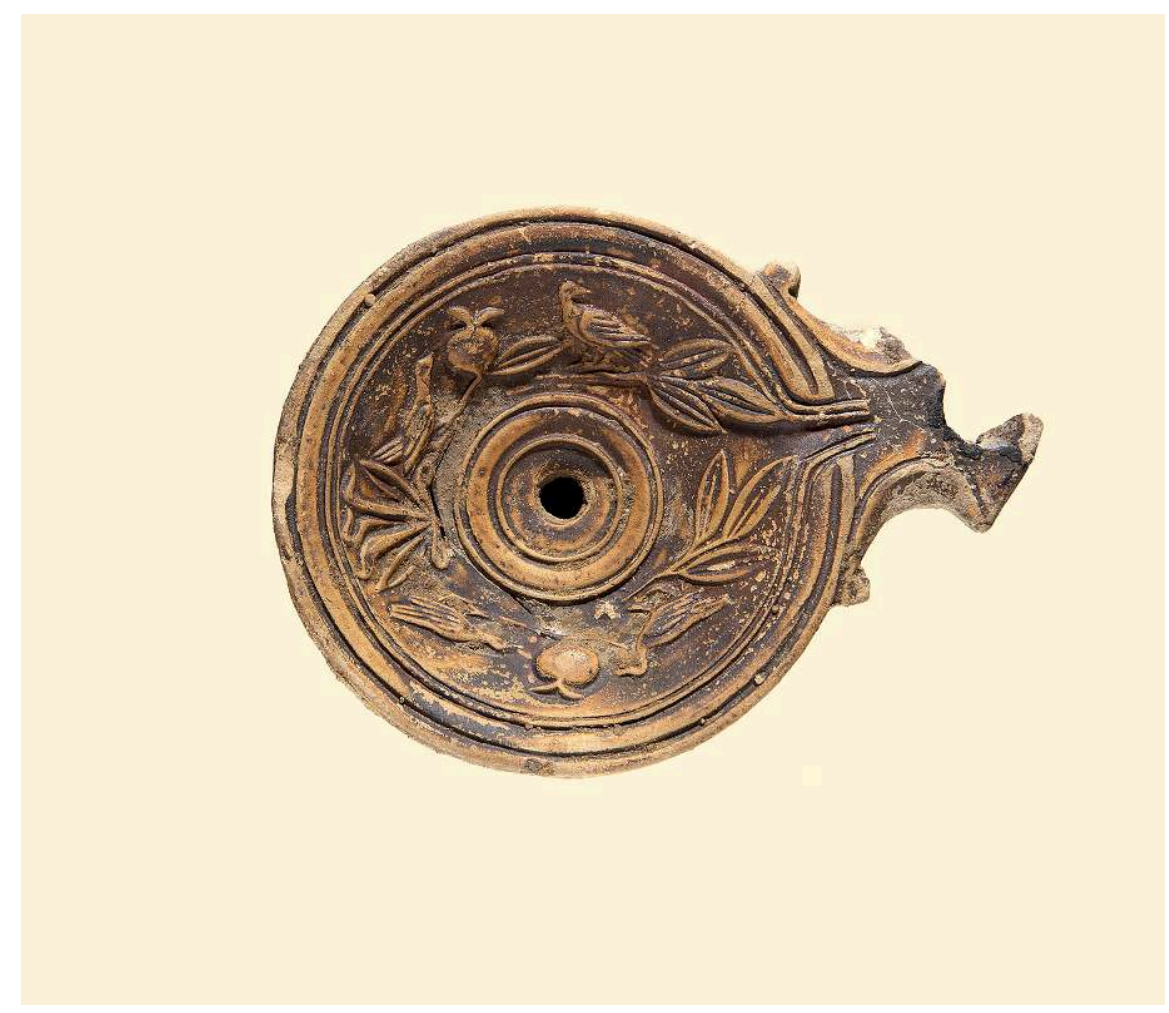

(C) Ifao. 17125_2020_NDMPM_001 
Fig. 13. Amulettes en faïence. III $\mathrm{e}$ s. av. J.-C. (Ihab Mohamed Ibrahim).
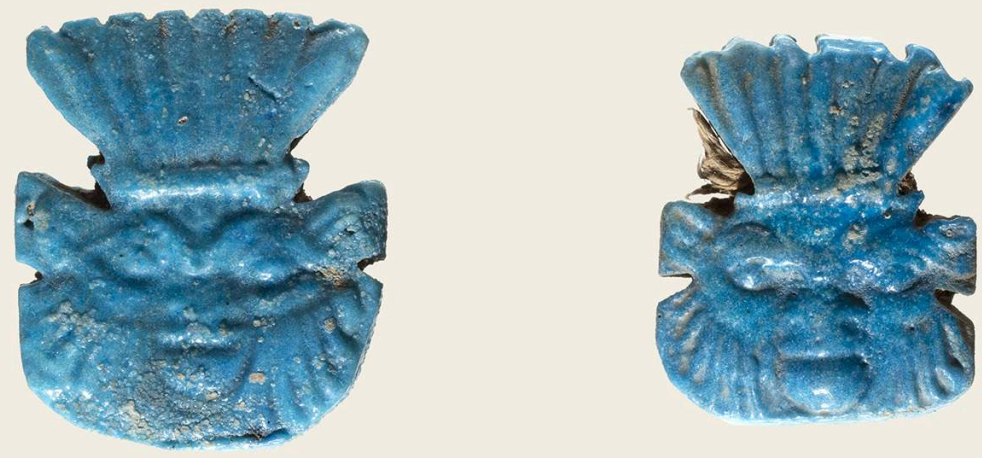

C Ifao, 17125_2020_NDMPM_002

Fig. 14. Bonnet en laine pour nourrisson ou enfant en bas-âge. $\|^{e}$ s. apr. J.-C. (Ihab Mohamed Ibrahim).

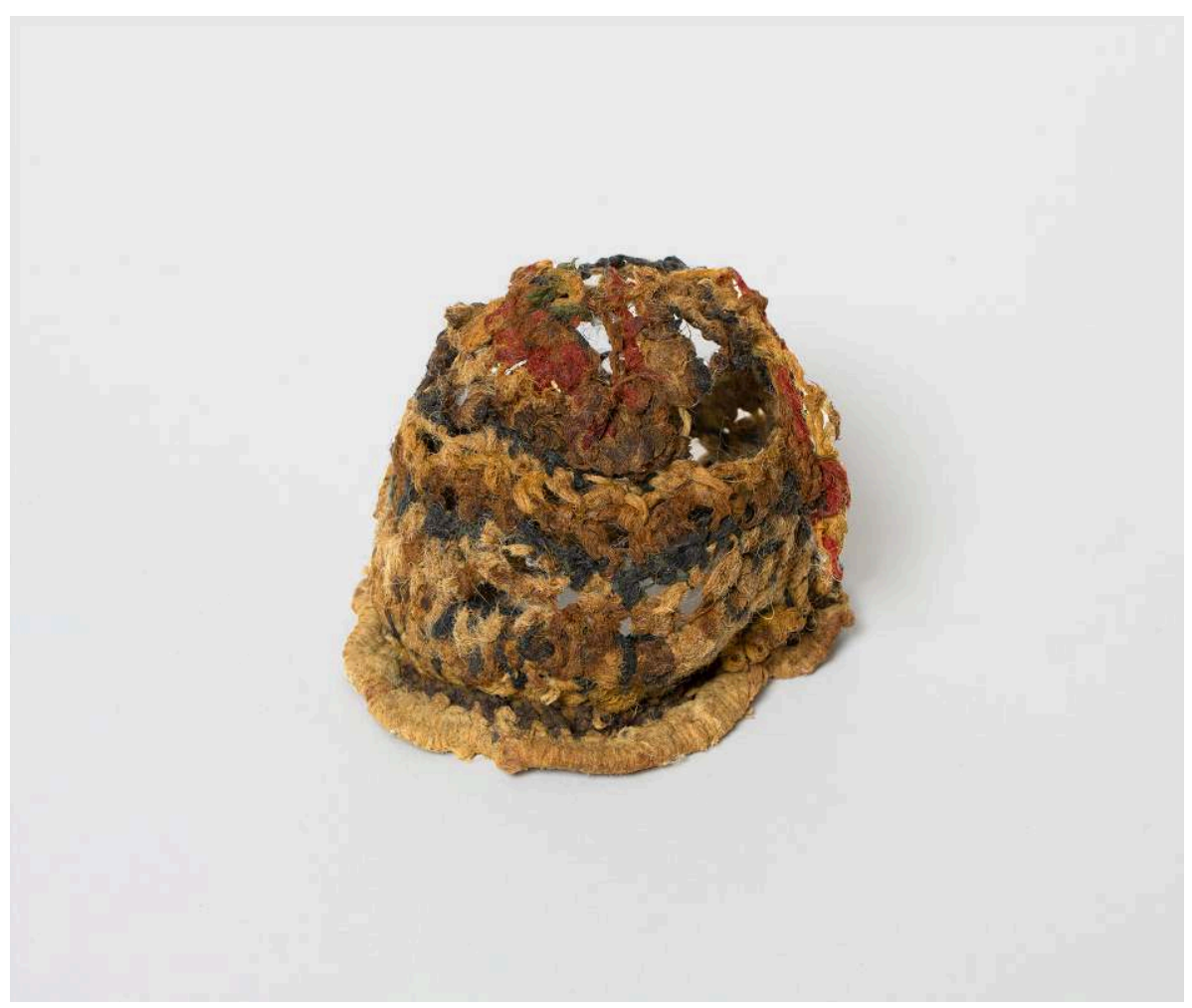

(C) Ifao. 17125_2020_NDMPM_003 
trouvailles venant d'être décrites, plus ou moins intéressantes pour une raison ou l'autre, il convient d'ajouter quelques pièces que nous pourrions qualifier d'uniques: un dé en calcaire, une souris en bois et une curieuse représentation en pierre du dieu Sobek.

Le dé, extrait de la grosse couche de sable, gravats et détritus qui recouvrait les restes du pyrgos C2200-III et contenait du matériel remontant au $\mathrm{I}^{\mathrm{er}}$ et $\mathrm{au} \mathrm{II}^{\mathrm{e}}$ s. apr. J.-C., est un petit cube de 12-13 $\mathrm{mm}$ de côté. Sur cinq faces sont creusés des trous ronds et peu profonds, noircis à l'encre, indiquant les chiffres habituels; sur la sixième, en revanche, est représenté un crocodile allongé sur un autel, tête dressée et la queue retombant droite à l'arrière. La gravure pourrait être le signe hiéroglyphique du «crocodile sur l'autel ${ }^{28}$, ou bien représenter Sobek selon une iconographie assez diffusée ${ }^{29}$; mais, quoi qu'il en soit, elle n'est pas courante sur les dés à jouer utilisés au quotidien ${ }^{30}$. Alors, devons-nous supposer que l'objet n'est pas un dé ? La figure du crocodile étant réalisée en creux, nous pourrions penser que la pièce est un moule cubique utilisé pour fabriquer des tablettes en argile historiées, comme celui des Musées royaux de Bruxelles publié dans NACHTERGAEL 1998, p. 159-177. Mais cette interprétation est exclue, car les moules cubiques ont des arêtes de $9-20 \mathrm{~cm}$ et portent sur toutes leurs faces des images de divinités ou d'entités en rapport avec les divinités (autels, victimes, couteaux de sacrifice) et non des trous correspondant à des chiffres ${ }^{31}$. De même, nous ne pouvons penser à un objet tel que l'hexaèdre du Petrie Museum inv. UC 38176, où sur les six faces sont gravés les noms en hiéroglyphe de six divinités (Osiris, Isis, Nephthys, Horus, Horus de Behedet, Hathor) ${ }^{32}$. Sur notre pièce, en effet, seule une face porte une image pouvant représenter le hiéroglyphe du «crocodile sur l'autel», tandis que sur les autres sont creusés les trous des chiffres. Par conséquent, mieux vaut considérer que notre pièce était un dé à jouer, où l'image de Sobek représentait le chiffre le plus haut, à savoir le 6, en même temps qu'elle était un appel au dieu, dont l'intervention apporterait le meilleur résultat. Cette interprétation paraît vraisemblable, mais, en l'absence de parallèles dans le mobilier connu et d'informations dans la documentation écrite, nous ne pouvons garantir son exactitude.

La souris en bois, longue de $6 \mathrm{~cm}$, provient d'une strate datée de la fin du II $^{\mathrm{e}}$ ou du début $\mathrm{du} \mathrm{I}^{\mathrm{er}}$ s. av. J.-C. (fig. 15). Il s'agit d'une statuette représentant le rongeur d'une façon très réaliste, mais sur laquelle certains détails sont difficiles à interpréter. Par exemple, une rainure large de presque $1 \mathrm{~cm}$ est taillée sous le ventre de l'animal, du postérieur à l'extrémité des pattes avant. En outre, plusieurs orifices percent le bois : un au sommet de la tête de la souris, un autre sous la mandibule, un troisième sur le dos, un quatrième dans l'arrière-train et deux autres dans les pattes arrière. De toute évidence, dans le trou de l'arrière-train était introduite la queue, probablement mobile, et aujourd'hui manquante. Dans ceux des pattes l'on introduisait peut-être l'essieu de deux petites roues, ou un pivot qui permettait à la souris de se dresser, à la manière des rats. Mais quelle était la fonction des autres ? Et à quoi servait la rainure du dessous ? Avant l'étude détaillée de l'objet, ces questions restent en suspens et la destination de la souris demeure incertaine. Frappés par la finesse du travail, nous pourrions imaginer que la sculpture était le décor original d'un meuble, d'une boîte, ou d'un couvercle. Mais c'était plus probablement un joli jouet, à l'exemple de la souris plus grossière du Fitzwilliam Museum de Cambridge inv. 4594-1943 ${ }^{33}$. 
Fig. 15. Souris en bois. $\|^{\mathrm{e}} \mathrm{I}^{\mathrm{er}}$ s. av. J.-C. (Ihab Mohamed Ibrahim, Fr. Cetera).

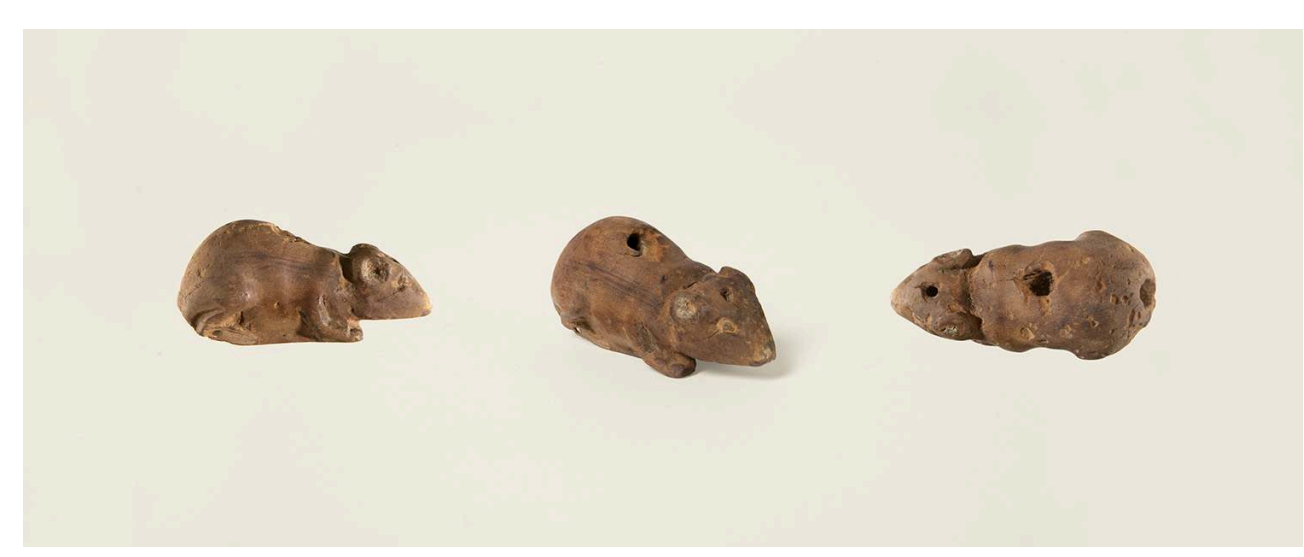

(c) Ifao. 17125_2020_NDMPM_004

La représentation de Sobek est sculptée dans un bloc de calcaire haut de $45 \mathrm{~cm}$, large de $14 \mathrm{~cm}$, épais de $8,5 \mathrm{~cm}$ à la base et de $11 \mathrm{~cm}$ à un tiers de sa hauteur (fig. 16). Elle était remployée comme matériau de construction dans le muret entourant la fosse d'arbre $\mathrm{du} \mathrm{II}^{\mathrm{e}} \mathrm{s}$. av. J.-C. retrouvée sous la maison C3200-III E. Cette utilisation n'est pas surprenante, car il suffit de jeter un coup d'œil sur la sculpture pour remarquer qu'elle est inachevée et qu'elle ne suit ni les canons de l'art égyptien, ni ceux de l'art grec. L'artiste, que nous qualifierons plutôt d'artisan, voulait représenter Sobek selon une iconographie traditionnelle: avec une tête de crocodile et un corps humain, debout, tenant l'ânkh d'une main et un sceptre de l'autre et coiffé d'une perruque tripartite surmontée du disque solaire. Mais, en exécutant son œuvre, il a traité le sujet en partie comme s'il réalisait une stèle, en partie comme s'il sculptait une statue en ronde-bosse. Il a aligné les pieds l'un derrière l'autre, a représenté le torse tourné vers le spectateur, le pan gauche de la perruque disposé de côté et le disque solaire parallèle au museau du crocodile, comme cela est visible sur les stèles. En revanche, il a sculpté en ronde-bosse la moitié gauche de la tête, le bout du museau, le bras portant l'ânkh et le bras tenant le sceptre, qui passe devant la poitrine. En même temps, il a réalisé le pan droit de la perruque retombant à plat sur la poitrine, comme s'il était vu de face. Il a donc réalisé son sujet à partir de points de vue différents, à savoir de profil et de face, et a mêlé deux techniques, celle du haut-relief et celle de la ronde-bosse. Malheureusement, il n'a pas achevé son œuvre, soit parce qu'il s'est aperçu avoir commis des erreurs, soit car il a compris que son œuvre n'aurait jamais satisfait les goûts de sa clientèle. Ainsi la sculpture a été mise au rebut et a été placée au sein des briques du muret construit autour de l'arbre. 
Fig. 16. Statue de Sobek en calcaire. $\|^{\mathrm{e}} \mathrm{s}$. av. J.-C. (Ihab Mohamed Ibrahim, Fr. Cetera).

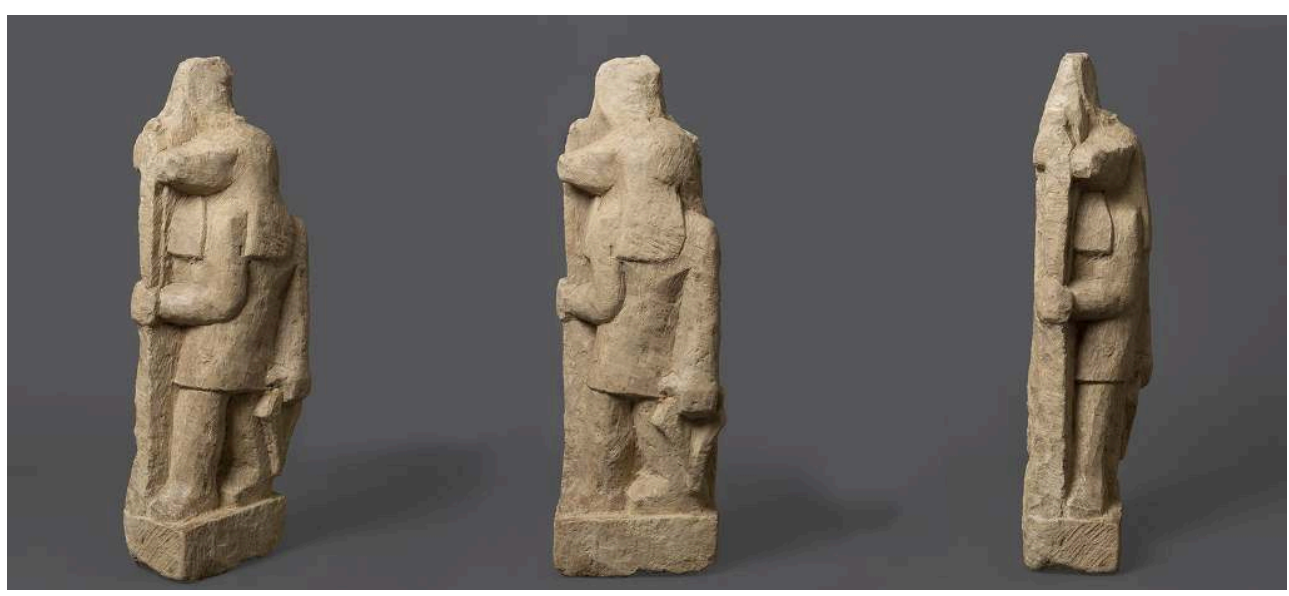

(C) Ifao. 17125_2020_NDMPM_005 amphores, tessons et papyrus, datés du milieu du $\mathrm{III}^{\mathrm{e}} \mathrm{s}$. av. J.-C. au milieu du $\mathrm{II}^{\mathrm{e}} \mathrm{s}$. de
notre ère. En ne comptant que les pièces susceptibles d'être publiées, nous en avons environ 200 : un ostracon hiéroglyphique et quelques papyrus hiératiques; une dizaine de dipinti sur amphore, 30 ostraca et près de 40 papyrus écrits en démotique; plus de 40 dipinti, une trentaine d'ostraca et environ 40 papyrus avec des textes grecs.

Quoique peu nombreuses, les pièces en hiéroglyphe et hiératique sont d'une importance non négligeable, parce qu'elles sont l'indice de la proximité d'un temple, à savoir celui du dieu dénommé Tefresudj(ty ?). Les textes démotiques, en majorité, ne sont pas différents de ceux récoltés dans les environs de 1989 à 1995 et en 2019. Néanmoins, 4 ostraca, remontant au $\mathrm{III}^{\mathrm{e}} \mathrm{s}$. av. J.-C. et retrouvés dans une couche de détritus non loin l'un de l'autre, doivent être mis en évidence, parce qu'ils ont une caractéristique les distinguant des milliers d'exemplaires récupérés auparavant: ils sont percés dans un angle et l'un d'eux conserve encore une ficelle longue d'une quinzaine de centimètres, qui passe dans le trou, est nouée aux extrémités et forme un anneau. Nous pouvons supposer qu'il en était de même pour les autres exemplaires, dont les trous n'ont plus de ficelle. D'après un premier examen, les tessons semblent porter les noms d'une ou deux personnes et, au moins dans un cas, une date ${ }^{34}$. Il est donc vraisemblable que les quatre ostraca percés servaient d'étiquettes et qu'ils étaient attachés par la ficelle à des marchandises ou à des objets pour en indiquer la propriété. Si nous acceptons cette interprétation, nous pouvons même facilement expliquer pourquoi deux des ostraca sont des palimpsestes: lorsqu'il fallait une nouvelle étiquette, au lieu de prendre la peine de percer un nouveau tesson, l'on préférait en recycler un déjà prêt, en effaçant le texte obsolète et en écrivant un autre nom audessus.

Parmi le matériel grec, les dipinti ne diffèrent pas de ceux édités dans LITINAS 2008. De même, les papyrus, qui portent tous des textes documentaires, quand ils sont datés de la période ptolémaïque, ressemblent à ceux récoltés depuis 1994 dans le grand dépotoir à l'est du temple de Soknebtynis; lorsqu'ils remontent à l'époque romaine, ils sont comparables à ceux récupérés dans l'habitat à partir de 1899. Les ostraca, enfin, ainsi que ceux recueillis en 2019 dans le même secteur du village, conservent des listes de

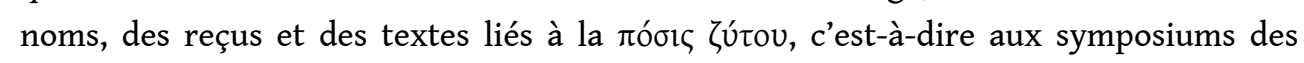

Bulletin archéologique des Écoles françaises à l'étranger , Égypte 
associations religieuses et professionnelles. Les reçus enregistrent des paiements de

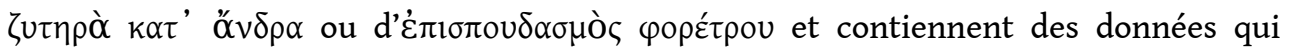

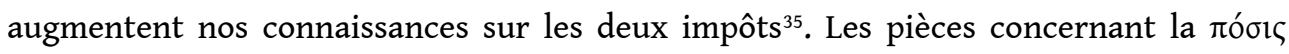

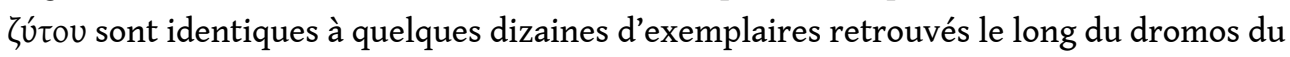
temple de Soknebtynis à partir de la campagne de l'année $2000^{36}$. Ils confirment, donc, que près du « dromos de Tefresudj(ty?) » étaient organisées des cérémonies comparables à celles se déroulant près de l'autre allée sacrée, comme nous l'avions pensé en 2019, lorsque les premiers documents de ce type sont apparus dans les bâtiments longeant la grande rue.

\section{BIBLIOGRAPHIE}

Les recueils de papyrus et d'ostraca sont cités avec les sigles de John F. Oates et al., Checklist of Editions of Greek, Latin, Demotic and Coptic Papyri, Ostraca and Tablets, BASP-Suppl. 9, 2001 et ses mises à jour sur le site https://scriptorium.lib.duke.edu/papyrus/texts/clist.html.

ADRIANI 1940

Achille Adriani, Annuaire du Musée gréco-romain d'Alexandrie (1935-1939), Alexandrie, Société de publications égyptiennes, 1940.

ANDREWS 1994

Carol Andrews, Amulets of Ancient Egypt, Londres, The British Museum Press, 1994.

BAILEY 2008

Donald Michael Bailey, Catalogue of the Terracottas in the British Museum, vol. 4 : Ptolemaic and Roman Terracottas from Egypt, Londres, The British Museum Press, 2008.

BRECCIA 1934

Evaristo Breccia, Terrecotte figurate greche e greco-egizie del Museo di Alessandria, vol. 2, Monuments de l'Égypte gréco-romain 2/2, Bergame, Istituto italiano d'arti grafiche, 1934.

BUSSIÈRE, WOHL 2017

Jean Bussière, Birgitte Lindros Wohl, Ancient Lamps in the J. Paul Getty Museum, Los Angeles, J. Paul Getty Museum Publications, 2017.

DUNAND 1979

Françoise Dunand, Religions populaire en Égypte romaine. Les terres cuites isiaques du Musée du Caire, EPRO 76, Leyde, Brill, 1979.

DUNAND 1990

Françoise Dunand, Catalogue des terres cuites gréco-romaines d'Égypte, Paris, Réunion des musées nationaux, 1990.

FITTÀ 1998

Marco Fittà, Spiele und Spielzeug in der Antike. Unterhaltung und Vergnügen im Altertum, Stuttgart, Konrad Theiss Verlag, 1998.

FROSCHAUER 2004

Harald Froschauer, « Brett- und Würfelspiele als traditionelle Freitvergnügungen », in Harald 
Froschauer, Hermann Harrauer (éd.), Spiel am Nil. Unterhaltung im Alten Ägypten: [Sonderausstellung im Papyrusmuseum der Österreichischen Nationalbibliothek], Nilus 10, Vienne, Phoibos Verlag, 2004, p. 23-34.

GALLAZZI 1997

Claudio Gallazzi, « Due campagne di scavo a Umm-el-Breigât (Tebtynis): 1995 e 1996 », Acme 50/3, 1997, p. 15-30.

GALLAZZI 1998

Claudio Gallazzi, « Lo scavo di una discarica a Umm-el-Breigât (Tebtynis), ovvero, le sorprese del pattume », NAC 27, 1998, p. 185-207.

GALLAZZI 2003

Claudio Gallazzi, « La prima campagna di Vogliano in Egitto. Gli scavi a Tebtynis e gli acquisti di papiri », in Claudio Gallazzi, Luigi Lehnus (éd.), Achille Vogliano cinquant'anni dopo, QuadAc 59, Milan, Cisalpino, 2003, p. 131-195.

\section{GALLAZZI 2005}

Claudio Gallazzi, « Umm-el-Breigât (Tebtynis) : 2002 », ASAE 79, 2005, p. 107-114.

GALLAZZI 2011

Claudio Gallazzi, « Umm-el-Breigât (Tebtynis). Campagne di scavo 2009-2010 », RISE 5, 2011, p. 109-130.

GALLAZZI 2020

Claudio Gallazzi, « Umm-el-Breigât (Tebtynis): campagna di scavo 2019 », RISE 8, 2020, p. 123-150. GALLAZZI, HADJI-MINAGLOU 2000

Claudio Gallazzi, Gisèle Hadji-Minaglou, Tebtynis I. La reprise des fouilles et le quartier de la chapelle d'Isis Thermouthis, FIFAO 42, Le Caire, Ifao, 2000.

GALLAZZI, HADJI-MINAGLOU 2010

Claudio Gallazzi, Gisèle Hadji-Minaglou, « Tebtynis », in Béatrice Midant-Reynes, Sylvie Denoix (éd.), « Travaux de l'Institut français d'archéologie orientale 2009-2010 », BIFAO 110, 2010, p. 303-477, en partic. p. 365-370.

GALLAZZI, HADJI-MINAGLOU 2020

Claudio Gallazzi, Gisèle Hadji-Minaglou, « Tebtynis », Bulletin archéologique des écoles françaises à l'étranger, 2020, article en ligne sur OpenEdition Journals, https://journals.openedition.org/ baefe/1075, consulté le 31 mai 2021.

GARDINER 1927 (éd. 1957)

Alan H. Gardiner, Egyptian Grammar: being an introduction to the study of hieroglyphs (1927), Oxford, Griffith Institute, Ashmolean Museum, 1957 (3éd.).

GRIMAL 1997

Nicolas Grimal, « Travaux de l'Institut français d'archéologie orientale en 1996-1997 », BIFAO 97, 1997, p. 313-429.

HADJI-MINAGLOU 2007

Gisèle Hadji-Minaglou, Tebtynis IV. Les habitations à l'est du temple de Soknebtynis, FIFAO 56, Le Caire, Ifao, 2007.

HADJI-MINAGLOU 2014

Gisèle Hadji-Minaglou, « Les maisons-tours de Tebtynis », in Séverine Marchi (éd.), Les maisonstours en Égypte durant la Basse Époque, les périodes ptolémaïque et romaine. Actes de la table-ronde de Paris, Université Paris-Sorbonne (Paris IV), 29-30 novembre 2012, NeHet 2, 2014, p. 33-56. 
KOCKELMANN 2017

Holger Kockelmann, Der Herr der Seen, Sümpfe und Flussläufe: Untersuchungen zum Gott Sobek und den ägyptischen Krokodilgötter-Kulten von den Anfängen bis zur Römerzeit, ÄgAbh 74, Wiesbaden, Harrassowitz, 2017.

KOSACK 1974

Wolfgang Kosack, Alltag im alten Ägypten: Aus der Ägyptensammlung des Museums für Völkerkunde der Stadt Freiburg im Breisgau, Veröffentlichungen des Museums für Völkerkunde 1, Fribourg-enBrisgau, Städtische Museen, 1974.

LITINAS 2008

Nikos Litinas, Tebtynis III: Vessel's Notations from Tebtynis, FIFAO 55, Le Caire, Ifao, 2008.

MATHIEU 2003

Bernard Mathieu, « Travaux de l'Institut français d'archéologie orientale en 2002-2003 », BIFAO 103, 2003, p. 487-664.

Milano in Egitto 2017

Milano in Egitto. Gli scavi di Achille Vogliano nel Fayum, catalogue d'exposition, Civico Museo Archeologico di Milano, 17 mai - 15 décembre 2017, Busto Arsizio, Nomos Edizioni, 2017.

NACHTERGAEL 1989

Georges Nachtergael, « Le chameau, l’âne et le mulet en Égypte gréco-romaine. Le témoignage des terres cuites », ChronEg 64, 1989, p. 287-336.

NACHTERGAEL 1998

Georges Nachtergael, «Un sacrifice en l'honneur de "Baubo" : scènes figurées sur un moule cubique de l'Égypte romaine », in Willy Clarysse, Antoon Schoors, Harco Willems (éd.), Egyptian Religion: The Last Thousand Years. Studies Dedicated to the Memory of Jan Quaegebeur, vol. 1, OLA 84, Louvain, Peeters, 1998, p. 159-177.

PETRIE 1917

William Matthew Flinders Petrie, Tools and Weapons illustrated by the Egyptian Collection in University College, London, and 2000 Outlines from other Sources, BSAE 30, Londres, British School of Archaeology in Egypt, 1917.

PETRIE 1927

William Matthew Flinders Petrie, Objets of Daily Use, BSAE 42, Londres, British School of Archaeology in Egypt, 1927.

REISNER 1907

George Andrew Reisner, Catalogue général des antiquités égyptiennes du Musée du Caire. N $N^{\circ 2} 5218-6000$ et 12001-12527: Amulets I, Le Caire, Ifao, 1907.

REISNER 1958

George Andrew Reisner, Catalogue général des antiquités égyptiennes du Musée du Caire.

$N^{o s}$ 12528-13595: Amulets II, Le Caire, Ifao, 1958.

REITER 2005

Fabian Reiter, « Symposia in Tebtynis - Zu den griechischen Ostraka aus den neuen Grabungen », in Sandra Lippert, Maren Schentuleit (éd.), Tebtynis und Soknopaiu Nesos: Leben im römerzeitlichen Fajum, Akten des Internationalen Symposions von 11. bis 13. Dezember 2003 in Sommerhausen bei Würzburg, Wiesbaden, Harrassowitz, 2005, p. 131-140.

TAIT 1998

John Tait, « Dicing with the Goods », in Willy Clarysse, Antoon Schoors, Harco Willems (éd.), 
Egyptian Religion: The Last Thousand Years. Studies Dedicated to the Memory of Jan Quaegebeur, vol. 1, OLA 84, Louvain, Peeters, 1998, p. 257-264.

VERNUS 2005

Pascal Vernus, « Souris », in Pascal Vernus, Jean Yoyotte, Bestiaire des pharaons, Paris, Perrin, 2005, p. 628-629.

WALLACE 1938

Sherman LeRoy Wallace, Taxation in Egypt from Augustus to Diocletian, Princeton, Princeton University Press, 1938.

VAN DE WALLE 1972

Baudouin van de Walle, « Rś-w $\underline{d} 3$ comme épithète et comme entité divine », ZÄS 98, 1972, p. 140-149.

WILFONG, FERRARA 2014

Terry G. Wilfong, Andrew W.S. Ferrara, « Karanis Revealed: Artefacts from the Exhibition », in Terry G. Wilfong, Andrew W.S. Ferrara (éd.), Karanis Revealed: Discovering the Past and Present of a Michigan Excavation in Egypt, catalogue d'exposition, Kelsey Museum of Archaeology, Ann Arbor, 6 septembre - 16 décembre 2011, 27 janvier - 6 mai 2012, Kelsey Museum Publication 7, Ann Arbor, Kelsey Museum of Archaeology, 2014.

WILLEMS, CLARYSSE 2000

Harco Willems, Willy Clarysse, Les Empereurs du Nil, catalogue d'exposition, Musée Gallo-romain, Tongres, 25 septembre - 6 février 2000, Louvain, Paris, Peeters, 2000.

\section{NOTES}

1. Les travaux réalisés en 2019 dans ces deux secteurs sont décrits dans GALLAZzI, HADJIMINAGLOU 2020, p. 3-21, § 5-35, et GALLAZZI 2020, p. 125-137.

2. Il est pour le moment impossible de dire quel était le dieu adoré dans le temple, car

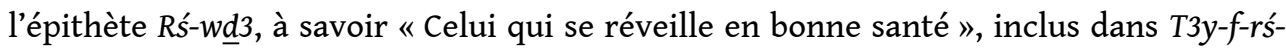
$w \underline{\text { } 3}$ (Tefresudj ou Tefresuty) est attribué à Osiris et Min, tous deux mentionnés dans des textes récupérés à Tebtynis, aussi bien qu'à Amon et Ptah: cf. VAN DE WALLE 1972, p. $140-149$.

3. Cf. GALLAZZI, HADJI-MINAGLOU 2000, p. 37-127, et HADJI-MINAGLou 2007, p. 9-164. Le tracé de la rue apparaît sur les plans publiés dans GALLAZZI, HADJI-MINAGLOU 2000, p. 40, et HADJIMINAGLOU 2007, p. 3-6.

4. Cf. GALLAZZI, HADJI-MiNAGLOU 2020, p. 6-15, § 8-30 ; GALLAZZI 2020, p. 127-135.

5. Les bâtiments mentionnés sont représentés sur les plans publiés dans GALLAZzI, HADJIMINAGLOU 2020, fig. 5-6 et GALLAZZI 2020, p. 144.

6. Sur ces deux constructions cf. GALLAZZI, HADJI-MINAGLOU 2020, p. 8, §11, et GALLAZZI 2020, p. 130-131, où les bâtiments portent encore les dénominations C4100-II W et C4100-II E, à présent abandonnées suite à une nouvelle interprétation des vestiges.

7. Cf. HADJI-MINAGLOU 2007, p. 139-147 ; HADJI-MINAGLOU 2014, p. 36-39.

8. Cf. GALlAZZI, HADJI-MiNAGLOU 2020, p. 10-11, § 19 ; GALLAZZI 2020, p. 132.

9. Pour le pyrgos 2800, construit dans la seconde moitié du $\mathrm{I}^{\mathrm{er}}$ s. av. J.-C., voir GRIMAL 1997, p. 360-361 ; GALLAZZI 1997, p. 23-25 ; HADJI-MINAGLOU 2014, p. 39-41. 
10. Cf. GALLAZZI, HADJI-MINAGLOU 2010, p. 366-368 ; GALLAZZI 2011, p. 112-114.

11. Cf. HADJI-MINAGLOU 2014, p. 49, fig. 17.

12. Cf. GALLAZZI, HADJI-MiNAGLOU 2020, p. 13-14, § 25 et fig. 7 ; GALLAZZi 2020, p. 128.

13. Cf. GALLAZZI 1998, p. 185-207.

14. Cf. GallaZZI, HAdjI-MinAGLOU 2020, p. 13, § 24 et fig. 7-8 ; GALLAZZi 2020, p. 128-129.

15. Cf. GALLAZZI, HADJI-MiNAGLOU 2020, p. 8-9, § 14 et fig. 6 ; GALLAZZI 2020, p. 131.

16. Cf. GALLAZZI, HADJI-MINAGLOU 2020, p. 11, § 20 et fig. 6 ; GALLAZZI 2020, p. 131-132 et plan IV.

17. Cf. MATHIEU 2003, p. 573 ; GALLAZZI 2005, p. 108.

18. Cf. GALLAZZI, HADJI-MINAGLOU 2020, p. 8-9, § 14 ; GALLAZZI 2020, p. 131.

19. Cf. GallazZi, hadji-MiNAGLOU 2020, p. 9 , § 15 et fig. 5 ; GALLAZZi 2020, p. 131.

20. Cf. GALLAZZI, HADJI-MINAGLOU 2020, p. 17, § 31 ; GALLAZZI 2020, p. 134.

21. Voir, par exemple, BRECCIA 1934, p. 5, $\mathrm{n}^{\circ} 2$, pl. 4, n ${ }^{\circ} 4$; ADRIANI 1940, p. 169, pl. LXII, $\mathrm{n}^{\circ} 2$; DUNAND 1990, p. 139, nº 368 ; BAILEY 2008, p. 101, pl. 63, nº 3351.

22. Cf. DUNAND 1979, p. 186, pl. XXXVI.

23. Cf. GALLAZZI 2003, p. 169.

24. Une lampe du même type, provenant d'Anatolie et datée de l'époque d'Auguste ou de Tibère, est décrite dans BUSSIÈre, wOHL 2017, p. 66-67, $\mathrm{n}^{\circ} 79$, ainsi que nous l'ont signalé Anna Południkiewicz et Iwona Zych, à qui nous adressons nos remerciements.

25. Des amulettes semblables sont publiées dans REISNER 1907, p. 184-187, $\mathrm{n}^{\text {os }} 12422-12438,12440-12447$, pl. XXIII, et WILFONG, FERRARA 2014, p. 57, $\mathrm{n}^{\text {os }} 21-22$.

26. Sur ce type d'amulette, voir ANDREWS 1994, p. 39-40, Des pièces presque identiques sont présentées dans REISNER 1958, p. 15, nºs 12643-12645, pl. III.

27. Cf. NACHTERGAEL 1989, p. 306-325 sur les bâts, paniers et fûts portés par des bêtes de somme.

28. Cf. GARDINER 1927 (éd. 1957), p. 475.

29. Cf. KOCKELMANN 2017, p. 114-118.

30. Pour les jeux de dés se reporter à FITTÀ 1998, p. 110-120, et FROSCHAUER 2004, p. 30-33.

31. Cf. NACHTERGAEL 1998, p. 160-161.

32. Cf. PETRIE 1927, p. 57, $\mathrm{n}^{\circ}$ 233, pl. XLIX, $\mathrm{n}^{\circ}$ 233, et TAIT 1998, p. 257-264. Selon les dires de Tait (1998, p. 261-264), cet hexaèdre aurait pu servir à des jeux ou à des pratiques oraculaires, ou bien aider au choix du nom des enfants, et aussi être utilisé dans des contextes funéraires. Aucune de ces hypothèses n'a trouvé de confirmation. De toute façon, il est impossible de voir comment notre dé aurait pu être utilisé pour obtenir la réponse d'un oracle, ou décider du nom à donner à un enfant, ou bien servir à quelque pratique concernant un défunt.

33. Voir WILLEMS, CLARYSSE 2000, p. 207, $\mathrm{n}^{\circ} 111$ pour la pièce de Cambridge, et VERNUS 2005, p. 629 pour les souris en bois données comme jouets aux enfants.

34. Nous remercions Gert Baetens et Willy Clarysse, qui ont aimablement examiné les photos des quatre ostraca et nous ont apporté de précieuses suggestions. 


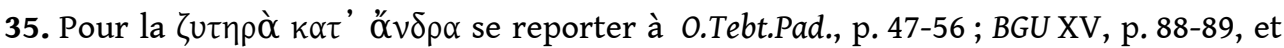

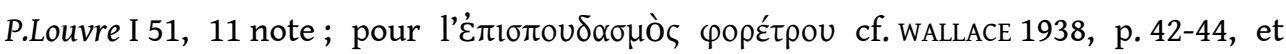
O.Tebt.Pad. 54, introduction.

36. Cf. REITER 2005, p. 131-140.

INDEX

Thèmes : IFAO

nature https://ark.frantiq.fr/ark:/26678/pcrtb1E0Dz7cSX

Année de l'opération : 2020

chronologie https://ark.frantiq.fr/ark:/26678/pcrtof7EHNsS2e

oeuvres https://ark.frantiq.fr/ark:/26678/pcrth1RXApKHOe

sujets https://ark.frantiq.fr/ark:/26678/pcrtux8pUagmKL, https://ark.frantiq.fr/ark:/26678/

pcrtbptj4SOA1W, https://ark.frantiq.fr/ark:/26678/pcrtb1E0Dz7cSX, https://ark.frantiq.fr/ark:/

26678/pcrtvskf7FZVME, https://ark.frantiq.fr/ark:/26678/pcrtEznNgQ3h63

lieux https://ark.frantiq.fr/ark:/26678/pcrtzcFLNEywS0, https://ark.frantiq.fr/ark:/26678/

pcrt6MfwpHpHsI

\section{AUTEURS}

\section{CLAUDIO GALLAZZI}

Papyrologue, Università degli studi di Milano

GISÈLE HADJI-MINAGLOU

Archéologue-architecte, Ifao 